\title{
Tetracycline hypersensitivity of an ezrA mutant links GalE and TseB (YpmB) to cell division
}

\section{OPEN ACCESS}

Edited by:

Jaan Männik,

University of Tennessee, USA

Reviewed by:

Akos T. Kovacs,

Friedrich Schiller

University of Jena, Germany

Mariana Pinho,

Instituto de Tecnologia Química e Biológica, Portugal

*Correspondence:

Pamela Gamba

Centre for Bacterial Cell Biology,

Institute for Cell and Molecular

Biosciences, Newcastle University,

Richardson Road, NE2 4AX,

Newcastle upon Tyne, UK

pamelagamba@gmail.com;

Leendert W. Hamoen,

Bacterial Cell Biology, Swammerdam

Institute for Life Sciences, University of

Amsterdam, Science Park 904, 1098

$\mathrm{XH}$, Amsterdam, Netherlands

I.w.hamoen@uva.nl

Specialty section:

This article was submitted to Microbial Physiology and Metabolism,

a section of the journal

Frontiers in Microbiology

Received: 11 February 2015 Accepted: 08 April 2015

Published: 22 April 2015

Citation:

Gamba P, Rietkötter E, Daniel RA and Hamoen LW (2015) Tetracycline hypersensitivity of an ezrA mutant links GalE and TseB (YpmB) to cell division.

Front. Microbiol. 6:346.

doi: 10.3389/fmicb.2015.00346

\author{
Pamela Gamba $^{1 *}$, Eva Rietkötter ${ }^{1}$, Richard A. Daniel ${ }^{1}$ and Leendert W. Hamoen ${ }^{1,2 *}$ \\ 'Centre for Bacterial Cell Biology, Institute for Cell and Molecular Biosciences, Newcastle University, Newcastle upon Tyne, \\ UK, ${ }^{2}$ Bacterial Cell Biology, Swammerdam Institute for Life Sciences, University of Amsterdam, Amsterdam, Netherlands
}

Cell division in bacteria is initiated by the polymerization of FtsZ into a ring-like structure at midcell that functions as a scaffold for the other cell division proteins. In Bacillus subtilis, the conserved cell division protein EzrA is involved in modulation of Z-ring formation and coordination of septal peptidoglycan synthesis. Here, we show that an ezrA mutant is hypersensitive to tetracycline, even when the tetracycline efflux pump TetA is present. This effect is not related to the protein translation inhibiting activity of tetracycline. Overexpression of FtsL suppresses this phenotype, which appears to be related to the intrinsic low FtsL levels in an ezrA mutant background. A transposon screen indicated that the tetracycline effect can also be suppressed by overproduction of the cell division protein ZapA. In addition, tetracycline sensitivity could be suppressed by transposon insertions in galE and the unknown gene ypmB, which was renamed tse $B$ (tetracycline sensitivity suppressor of $\underline{e} z \mathrm{~A} A$ ). GalE is an epimerase using UDP-glucose and UDP-N-acetylglucosamine as substrate. Deletion of this protein bypasses the synthetic lethality of zapA ezrA and sepF ezrA double mutations, indicating that GalE influences cell division. The transmembrane protein TseB contains an extracytoplasmic peptidase domain, and a GFP fusion shows that the protein is enriched at cell division sites. A tseB deletion causes a shorter cell phenotype, indicating that TseB plays a role in cell division. Why a deletion of ezrA renders $B$. subtilis cells hypersensitive for tetracycline remains unclear. We speculate that this phenomenon is related to the tendency of tetracycline analogs to accumulate into the lipid bilayer, which may destabilize certain membrane proteins.

\section{Keywords: FtsZ, EzrA, tetracycline, FtsL, GalE, Bacillus subtilis}

\section{Introduction}

Division of a bacterial cell involves the coordinated action of several proteins that localize at midcell and assemble in a multiprotein complex known as the divisome. The most crucial component of the division machinery is FtsZ, a structural homolog of eukaryotic tubulin (Lowe and Amos, 1998), which polymerizes into a ring-like structure at midcell when cell division is initiated (Bi and Lutkenhaus, 1991; Peters et al., 2007). In B. subtilis, the Z-ring is tethered to the membrane by FtsA (Wang et al., 1997; Ma and Margolin, 1999) and SepF (Duman et al., 2013), and functions as a scaffold for all other division proteins (for review, see Adams and Errington, 2009). Bundling of FtsZ protofilaments is stimulated by both SepF and the conserved protein ZapA 
(Gueiros-Filho and Losick, 2002; Singh et al., 2008; Gundogdu et al., 2011; Pacheco-Gomez et al., 2013). Another conserved early cell division protein that binds to the Z-ring is EzrA, which will be discussed below. After the Z-ring has formed, the late cell division proteins are recruited (Gamba et al., 2009). The transmembrane proteins PBP 2B, FtsL, DivIB, and DivIC are interdependent for their recruitment to the Z-ring. PBP $2 \mathrm{~B}$ is the transpeptidase that introduces cross-links into septal peptidoglycan (Daniel et al., 1996). The exact function of FtsL, DivIB, and DivIC is unclear. FtsL is efficiently degraded by the regulatory protease RasP through intramembrane proteolysis (Bramkamp et al., 2006), and presumably plays a regulatory role because of its marked instability (Daniel et al., 2006). DivIC is also an unstable protein (Robson et al., 2002). DivIB might have a role in the regulation of FtsL and DivIC stability (Daniel et al., 2006).

Several proteins modulate the assembly of the Z-ring in space and time. In Bacillus subtilis, the Min proteins prevent cell division at newly formed cell poles by inhibiting FtsZ bundling (Dajkovic et al., 2008; Gregory et al., 2008), and by promoting disassembly of the divisome after division is completed (van Baarle and Bramkamp, 2010). The nucleoid occlusion protein Noc binds to specific DNA sequences and prevents Z-ring assembly over the nucleoid, thereby coordinating cell division with DNA segregation (Wu and Errington, 2004; Wu et al., 2009; Adams et al., 2015). Cell division also responds to the metabolic status of the cell. The glucosyltransferase UgtP, involved in the synthesis of lipoteichoic acids, has been shown to accumulate at septa and to inhibit cell division in a growth-rate dependent manner (Weart et al., 2007; Chien et al., 2012). Recently, a link to central carbon metabolism has been established with the discovery that pyruvate levels can affect Z-ring formation (Monahan et al., 2014).

The early cell division protein EzrA is conserved in low $\mathrm{G}+\mathrm{C}$ Gram-positive bacteria. The protein is anchored to the cell membrane by an $\mathrm{N}$-terminal transmembrane domain and has a large cytoplasmic C-terminal domain that binds to FtsZ (Levin et al., 1999; Haeusser et al., 2004). Initially, it was assumed that EzrA negatively regulates Z-ring formation since an ezrA mutant shows an increased frequency of Z-rings in fast growth rate conditions, and purified EzrA inhibits bundling of FtsZ protofilaments (Haeusser et al., 2004; Chung et al., 2007; Singh et al., 2007). However, the function of EzrA is more complicated. Cells lacking EzrA are significantly longer than wild-type cells because of a delay in constriction (Levin et al., 1999; Kawai and Ogasawara, 2006), and deletion of the positive Z-ring regulators zapA or sepF in an ezrA background causes a severe block in cell division (Gueiros-Filho and Losick, 2002; Hamoen et al., 2006). A recent crystallographic study suggested that EzrA forms large semi-circular structures that can hook FtsZ filaments to the cell membrane. The large curved EzrA structures show some homology to the spectrin proteins which connect actin filaments in eukaryotes (Cleverley et al., 2014). Another activity of EzrA is the recruitment of the major transglycosylase/transpeptidase PBP 1 from the lateral wall to the division site (Claessen et al., 2008; Tavares et al., 2008).

Here we describe a peculiar phenotype of an ezrA mutant, the hypersensitivity to the antibiotic tetracycline. Detailed analysis of this phenomenon revealed that this sensitivity is not related to the classical inhibitory effect of tetracycline on protein translation. We show that overexpression of FtsL can suppress the tetracycline effect, and low levels of this key cell division regulator might be the reason for the phenotype. Using an extensive transposon screen we identified two new genes, galE and $y p m B$, which suppress the tetracycline sensitivity of an ezrA mutant when deleted. Interestingly, the absence of the UDP-galactose epimerase GalE restores also the lethal cell division defects of a ezrA sepF or ezrA zapA double mutant. Since a transposon insertion in the unknown $y p m B$ gene suppresses the tetracycline induced defects of an ezrA mutant, the gene was renamed $t s e B$ (tetracycline sensitivity suppressor of $\underline{e} z r A$ ). TseB is a membrane protein with an extracellular protease domain, and is enriched at cell division sites. The absence of this protein causes a short cell phenotype, further suggesting a role in cell division.

\section{Materials and Methods}

\section{Bacterial Strains and Growth Conditions}

Strains and plasmids used in this study are listed in Table 1. $B$. subtilis strains were grown at $30^{\circ} \mathrm{C}$ or $37^{\circ} \mathrm{C}$ in Antibiotic medium no. 3 (PAB, Difco, or Oxoid), LB or competence medium (CM) (Hamoen et al., 2002). Agar (Bacteriological agar no. 1, Oxoid) was added to a final concentration of $1.5 \%$ to prepare solid media. When required, media were supplemented with $10 \mu \mathrm{g} / \mathrm{ml}$ tetracycline (unless stated otherwise), $5 \mathrm{mM} \mathrm{MgSO}_{4}, 22.5 \mu \mathrm{M}$ EDTA, $22.5 \mu \mathrm{M}$ phenanthroline, or $0.5 \mu \mathrm{g} / \mathrm{ml}$ anhydrotetracycline. If needed, xylose and IPTG were used as inducers at concentrations of $0.5-2 \%$, and $1 \mathrm{mM}$, respectively. Selection of transformants was performed on nutrient agar (Oxoid), supplemented when required with $10 \mu \mathrm{g} / \mathrm{ml}$ tetracycline, $5 \mu \mathrm{g} / \mathrm{ml}$ chloramphenicol, $50 \mu \mathrm{g} / \mathrm{ml}$ spectinomycin, $5 \mu \mathrm{g} / \mathrm{ml}$ kanamycin or $0.5 \mu \mathrm{g} / \mathrm{ml}$ erythromycin with $25 \mu \mathrm{g} / \mathrm{ml}$ lincomycin. E. coli strains were grown in $\mathrm{LB}$ at $37^{\circ} \mathrm{C}$ and used as cloning intermediates.

\section{Growth Assays on Agar Plates}

Frozen stocks were streaked out to single colonies on nutrient agar plates supplemented as required and grown overnight at $37^{\circ} \mathrm{C}$. To ensure an even distribution of cells on all the plates that had to be compared, fresh single colonies were picked and spread onto a short streak on a new nutrient agar plate. This primary streak was then crossed with a new sterile loop that was used to transfer the inoculum on a new plate and isolate single colonies. Then, for each of the other agar plates that had to be compared within the same experiment, the same procedure was repeated with a new sterile loop by crossing the primary streak on a different (yet adjacent) point.

\section{Plasmid and Strain Construction}

Molecular cloning, PCRs, and E. coli transformations were carried out by standard techniques. Oligonucleotides used in this study are listed in Table 2 . To construct plasmid pPG6(mGFP), a fragment of $504 \mathrm{bp}$ containing the $t s e B$ coding sequence was amplified from 168 chromosomal DNA with oligonucleotides PG77 and PG79, carrying the HindIII and XhoI restriction sites, respectively. The insert was then cloned into an equally cut pSG1729, resulting in plasmid pPG6. Plasmid pPG6 was then 
TABLE 1 | Strains and plasmids used in this study.

\begin{tabular}{|c|c|c|}
\hline Strain & Relevant features or genotype & $\begin{array}{l}\text { Construction, source } \\
\text { or reference }\end{array}$ \\
\hline \multicolumn{3}{|c|}{ B. subtilis } \\
\hline 168 & $\operatorname{trpC2}$ & Laboratory stock \\
\hline BSB1 & $\operatorname{trp}+$ & Nicolas et al., 2012 \\
\hline 1356 & zapA-yshB::tet & $\begin{array}{l}\text { Feucht and Errington, } \\
2005\end{array}$ \\
\hline 2020 & amyE::(P $\mathrm{P}_{x y l} \mathrm{I}^{-g f p m u t 1-f t s Z, s p c)}$ & J. Sievers (unpublished) \\
\hline 3362 & ezrA::tet & Hamoen et al., 2006 \\
\hline 3828 & $\begin{array}{l}\text { ftsL::pSG441 aphA-3 } P_{\text {spac-pbpB, }} \\
\text { amyE::cat } P_{x y \mid}-\Delta 30 \text {-ftsL }\end{array}$ & Bramkamp et al., 2006 \\
\hline 4077 & ylmBC::(erm Pspac-ylmD), ezrA::tet & Hamoen et al., 2006 \\
\hline 814 & $\begin{array}{l}\Delta f t s L-P s p a c-p b p B \text { kan, } \\
\text { amyE::Pxyl-HA-ftsL cat }\end{array}$ & $\begin{array}{l}\text { Daniel and Errington, } \\
2000\end{array}$ \\
\hline BG239 & thr-5, tet- 4 & $\begin{array}{l}\text { Wei and Bechhofer, } \\
2002\end{array}$ \\
\hline KS273 & aprE::Pspac-zapA spc & Surdova et al., 2013 \\
\hline LH28 & ezrA::cat & $\begin{array}{l}\text { L. Hamoen } \\
\text { (unpublished) }\end{array}$ \\
\hline SG82 & lacA::tet & Laboratory stock \\
\hline YK012 & CRK6000 ezrA::spc & $\begin{array}{l}\text { Kawai and Ogasawara, } \\
2006\end{array}$ \\
\hline YK204 & CRK6000 sepF::spc & Ishikawa et al., 2006 \\
\hline PG49 & ezrA::spc & YK012 DNA $\rightarrow 168$ \\
\hline PG100 & lacA::tet, ezra::cat & LH28 DNA $\rightarrow$ SG82 \\
\hline PG112 & tet-4 & $\begin{array}{l}5 \mathrm{~kb} \text { rps } J \text { region from } \\
\text { BG239 } \rightarrow 168\end{array}$ \\
\hline PG113 & tet- 4 , ezrA::spc & $\begin{array}{l}5 \mathrm{~kb} \text { rps } J \text { region from } \\
\text { BG239 } \rightarrow \text { PG49 }\end{array}$ \\
\hline PG116 & tet-4, ezrA::cat & $\begin{array}{l}5 \mathrm{~kb} \text { rps } J \text { region from } \\
\text { BG239 } \rightarrow \text { LH28 }\end{array}$ \\
\hline PG121 & ezrA::tet, tseB:TnYLB-1 kan & $\begin{array}{l}\text { pMarB integration into } \\
3362\end{array}$ \\
\hline PG126 & ezrA::tet, zapA-TnYLB-1- yshB kan & $\begin{array}{l}\text { pMarB integration into } \\
3362\end{array}$ \\
\hline PG129 & ezrA::tet, galE:TnYLB-1 kan & $\begin{array}{l}\text { pMarB integration into } \\
3362\end{array}$ \\
\hline PG135 & tseB:TnYLB-1 kan & PG121 DNA $\rightarrow 168$ \\
\hline PG140 & zapA-TnYLB-1- yshB kan & PG126 DNA $\rightarrow 168$ \\
\hline PG143 & galE:TnYLB-1 kan & PG129 DNA $\rightarrow 168$ \\
\hline PG149 & aprE::Pspac-zapA, spc & KS273 DNA $\rightarrow 168$ \\
\hline PG158 & sepF::spc & YK204 DNA $\rightarrow 168$ \\
\hline PG160 & ezrA::tet, amyE::: $P_{x y l}-\Delta 30 f t s L-c a t$ & 3828 DNA $\rightarrow 3362$ \\
\hline PG162 & ezrA::cat, aprE::Pspac-zapA spc & PG149 DNA $\rightarrow$ LH28 \\
\hline PG164 & $\begin{array}{l}\text { ezrA::cat, zapA-yshB::tet, } \\
P_{\text {spac-zapA }}\end{array}$ & 1356 DNA $\rightarrow$ PG162 \\
\hline PG209 & ezrA::tet, amyE::P $P_{x y l}{ }^{-g f p-f t s} Z s p c$ & 2020 DNA $\rightarrow 3362$ \\
\hline PG234 & galE::kan & This work \\
\hline PG235 & tseB::kan & This work \\
\hline PG238 & ezrA::tet, galE::kan & PG234 DNA $\rightarrow 3362$ \\
\hline PG239 & ezrA::tet, tseB::kan & PG235 DNA $\rightarrow 3362$ \\
\hline PG251 & galE::spc & This work \\
\hline PG252 & tseB::spc & This work \\
\hline PG294 & ezrA::tet, galE::kan, sepF::spc & YK204 DNA $\rightarrow$ PG238 \\
\hline
\end{tabular}

(Continued)
TABLE 1 | Continued

\begin{tabular}{|c|c|c|}
\hline Strain & Relevant features or genotype & $\begin{array}{l}\text { Construction, source } \\
\text { or reference }\end{array}$ \\
\hline PG296 & $\begin{array}{l}\text { galE::kan, y/mBC::(erm } \\
P_{\text {spac-ylmD), ezrA::tet }}\end{array}$ & 4077 DNA $\rightarrow$ PG238 \\
\hline PG305 & $\begin{array}{l}\Delta f t s L-P_{\text {spac }}-p b p B \text { kan, } \\
\text { amyE }:: P_{x y l}-H A-f t s L \text { cat, ezrA::tet }\end{array}$ & 3362 DNA $\rightarrow 814$ \\
\hline PG307 & $\begin{array}{l}\text { ezrA::cat, zapA-yshB::tet, } \\
\text { aprE::Pspac-zapA spc, galE::kan }\end{array}$ & PG234 DNA $\rightarrow$ PG164 \\
\hline PG325 & aprE::Pspac $-t s e B$ spc & $\mathrm{pPG} 16 \rightarrow 168$ \\
\hline PG327 & aprE::Pspac-galE spc & pPG18 $\rightarrow 168$ \\
\hline PG330 & tseB::kan, aprE::Pspac-tseB spc & PG325 DNA $\rightarrow$ PG235 \\
\hline PG332 & $\begin{array}{l}\text { ezrA::tet, galE::kan, } \\
\text { aprE::Pspac-galE spc }\end{array}$ & PG327 DNA $\rightarrow$ PG239 \\
\hline PG333 & $\begin{array}{l}\text { ezrA::tet, tseB::kan, } \\
\text { aprE::Pspac-tseB spc }\end{array}$ & PG325 DNA $\rightarrow$ PG239 \\
\hline PG718 & $\operatorname{trp}+$, amyE::P $P_{x y l}-g f p-t s e B s p c$ & $\begin{array}{l}\text { pPG6(mGFP) } \\
\text { integration into BSB1 }\end{array}$ \\
\hline PG742 & $\begin{array}{l}\Delta f t s L-P s p a c-p b p B \text { kan, } \\
\text { amyE::Pxyl-HA-ftsL cat, lacA::tet }\end{array}$ & SG82 DNA $\rightarrow 814$ \\
\hline \multicolumn{3}{|l|}{ E. coli } \\
\hline $\mathrm{DH} 5 \alpha$ & $\begin{array}{l}F^{-}, \text {p80lacZ } \Delta M 15, \\
\Delta(\text { lacZYAargF }) \cup 196, \text { recA1, endA1, } \\
\text { hsdR17, }\left(r_{K-}, m_{K+}\right), \text { phoA, } \\
\text { supE44, } \lambda^{-}, \text {thi- } 1, \text { gyrA96, relA1 }\end{array}$ & Invitrogen \\
\hline Plasmid & Relevant features or genotype & $\begin{array}{l}\text { Construction, source } \\
\text { or references }\end{array}$ \\
\hline pAPNC213 & bla aprE' spc lacl Pspac'aprE & Morimoto et al., 2002 \\
\hline pMarB & bla erm $P_{\text {ctc }}$ Himar1 kan (TnYLB-1) & Le Breton et al., 2006 \\
\hline pSG1729 & $\begin{array}{l}\text { bla amyE3' spc } P_{x y l} \text {-gfpmut1' } \\
\text { amyE5' }\end{array}$ & $\begin{array}{l}\text { Lewis and Marston, } \\
1999\end{array}$ \\
\hline pHT21 & kan & $\begin{array}{l}\text { Trieu-Cuot and } \\
\text { Courvalin, } 1983\end{array}$ \\
\hline pLOSS* & $s p c$ & Claessen et al., 2008 \\
\hline pPG6(mGFP) & $\begin{array}{l}\text { bla amyE3'spc } P_{x y \mid} \text { tseB-mgfpm } 1^{\prime} \\
\text { amyE5' }\end{array}$ & This work \\
\hline pPG16 & bla aprE' spc lacl Pspac tseB'aprE & This work \\
\hline pPG18 & bla aprE' spc lacl $P_{\text {spac galE'aprE }}$ & This work \\
\hline
\end{tabular}

Unless stated otherwise, all strains were made in the 168 wild type background. Genes responsible for resistance to antibiotics are abbreviated as follows: bla, ampicillin; cat, chloramphenicol; erm, erythromycin; kan, kanamycin; spc, spectinomycin; tet, tetracycline.

used for a quick change mutagenesis reaction with oligonucleotides HS410 and HS411 in order to introduce the A206K mutation in the GFP coding sequence to reduce protein dimerization. The resulting plasmid was verified by sequencing and named pPG6(mGFP).

Plasmids pPG16 and pPG18 were derived from pAPNC213, which was digested with EcoRI and BamHI, and ligated with PCR products digested with the same restriction enzymes. For plasmid pPG16, the $t s e B$ coding sequence, including the ribosome binding site, was amplified using oligonucleotides PG152 and PG159. For plasmid pPG18, the galE coding sequence and $70 \mathrm{bp}$ of the upstream region was amplified with oligonucleotides PG70 and PG161. 


\begin{tabular}{|c|c|c|}
\hline Name & $\begin{array}{l}\text { Restriction } \\
\text { site }\end{array}$ & Sequence $\left(5^{\prime}-3^{\prime}\right)$ \\
\hline HS410 & & СCTGTCCACACAATCTAAACTTTCGAAAGATCCC \\
\hline HS411 & & GGGATCTTCGAAAGTTAGATTGTGTGGACAGG \\
\hline $\mathrm{Km} 3$ & $\mathrm{BamHI}$ & GGGGGATCCAAGACGAAGAGGATGAAG \\
\hline $\mathrm{Km} 4$ & EcoRl & CCCGAATTCAGAGTATGGACAGTTGCG \\
\hline olPCR1 & & GCTTGTAAATTCTATCATAATTG \\
\hline OIPCR2 & & AGGGAATCATTTGAAGGTTGG \\
\hline OIPCR3 & & GCATTAATACTAGCGACGCC \\
\hline PG57 & & TGATGGTGCTCCAGAAGAAC \\
\hline PG58 & & ACAGAACCACGAACTGTAGG \\
\hline PG70 & EcoRl & GCCGAATTCTTATTCCGCACTCTTATACCCATT \\
\hline PG77 & HindllI & CGGAAGCTTTTAAGGCGTGATATIITGAGAA \\
\hline PG79 & Xhol & CGGCTCGAGATGAGAAAAAAAGCATTAATATTTACCG \\
\hline PG103 & $\mathrm{BamHI}$ & GACGGATCCCTTCTCACCTACGTACGATA \\
\hline PG120 & & TACCTTCCTGCAGCTGATTC \\
\hline PG121 & & GAGCAGCTTACTGGAATCTC \\
\hline PG122 & HindllII & GATCAGTAAGCTTGACGAATTAGGGGGAGTTCAAG \\
\hline PG128 & $\mathrm{BamHI}$ & GAAGGATCCCCTAAAAAATGACCTGTIT \\
\hline PG129 & & CTCCGTTCCTCCACTTGATG \\
\hline PG130 & EcoRl & ATAGAATTCGAATGGAGGCCTTCTCAATT \\
\hline PG131 & & ATGATGATCGCCCGCGAAAC \\
\hline PG134 & Ncol & GAGTCCATGGTCAGAGTATGGACAGTTGCG \\
\hline PG135 & Ncol & GATCCCATGGGACGAATTAGGGGGAGTTCAAG \\
\hline PG146 & $\mathrm{BamHI}$ & CCGAGGATCCAGGATGTACTTAAACGCTAACG \\
\hline PG149 & HindllI & GCCAAGCTTCAAGAGGACGCTTTATTCTTC \\
\hline PG152 & EcoRl & CGTCGAATTCTTAAGGCGTGATATTITTGAGAA \\
\hline PG159 & $\mathrm{BamHI}$ & АCCTGGATCCTCGGCCTTGCGCTGGATGAAGA \\
\hline PG161 & $\mathrm{BamHI}$ & GGCAGGATCCCTATTAATAAACGATTAAACTTC \\
\hline $\begin{array}{l}\text { Spc-pLoss- } \\
\text { Rev }\end{array}$ & EcoRl & GCAGCCGAATTCCAAGAGGACGCTTTATCTTC \\
\hline
\end{tabular}

Recognition sites for restriction enzymes are indicated in bold.

Genes were deleted by replacing their coding sequences with antibiotic resistance cassettes. Approximately $3 \mathrm{~kb}$ upstream and downstream of the coding sequence of the gene of interest were amplified. For deletion of galE, oligonucleotides PG128PG129 and PG130-PG131 were used. For tseB deletions, oligonucleotides PG120-PG135 and PG103-PG131 were used for a deletion with a kan cassette, while PG120-PG122 and PG103-PG121 were used to construct a deletion with a $s p c$ cassette. Relevant restriction sites were inserted into the primers. Ligation reactions were assembled with equimolar concentrations of each of the three PCR products, using about $1.5 \mu \mathrm{g}$ of each $3 \mathrm{~kb}$ product in a total volume of $40 \mu \mathrm{l}$. Competent cells of B. subtilis were transformed with half of each ligation reaction. Transformants were selected on antibiotic plates and verified by PCR. Antibiotic resistance cassettes were amplified from plasmids: kan from pHT21 (oligonucleotides km3-km4 for galE and km3-PG134 for tseB), spc from pLOSS* (oligonucleotides PG146 and spc-pLoss-Rev for gale, PG146-PG149 for tseB).

An N-terminal GFP fusion to tseB was constructed by transforming pPG6(mGFP) plasmid into strain BSB1, generating strain PG718. The integration was obtained by a double crossover recombination event between the amyE regions located on the plasmid and the chromosomal $a m y E$ gene of strain 168. Transformants were selected on nutrient agar plates containing spectinomycin. Correct integration into the amyE gene was tested and confirmed by lack of amylase activity upon growth on plates containing $1 \%$ starch.

\section{Microscopic Imaging}

Samples were mounted on microscope slides coated with a thin layer of $1.2 \%$ agarose. Images were acquired with a Zeiss Axiovert $200 \mathrm{M}$ or a Zeiss Axiovert 135 microscope coupled to a Sony Cool-Snap HQ cooled CCD camera (Roper Scientific), and using Metamorph imaging software (Universal Imaging). For membrane staining, cells were mounted on slides coated with $1 \%$ agarose supplemented with the membrane dye Nile Red $(0.1 \mu \mathrm{g} / \mathrm{ml}$, Molecular Probes) or by mixing $9 \mu \mathrm{l}$ of cells with $1 \mu \mathrm{l}$ of Nile Red solution $(12.5 \mathrm{mg} / \mathrm{ml})$ before spotting the sample on the agarose slide. Alternatively, cells were mixed with the membrane dye FM5-95 (Invitrogen), at a final concentration of $0.4 \mu \mathrm{g} / \mathrm{ml}$. Nucleoids were stained by adding DAPI $(0.02 \mu \mathrm{g} / \mathrm{ml}$, Sigma $)$ to the agarose slide. Images were analyzed and prepared for publication with ImageJ (http://rsb. info.nih.gov/ij/). For time-lapse microscopy, strain PG718 was grown in $\mathrm{CM}$ supplemented with $0.5 \%$ xylose at $30^{\circ} \mathrm{C}$ until cells reached exponential phase, and subsequently mounted onto a thin semisolid matrix made of CM supplemented with $0.5 \%$ xylose and $1.5 \%$ low-melting point agarose on a microscope slide. Slides were incubated in a temperature-controlled chamber $\left(30^{\circ} \mathrm{C}\right)$ on a Deltavision RT automated microscope (Applied Precision). Phase contrast and GFP images were taken every $10 \mathrm{~min}$.

\section{Screen for Tetracycline-Insensitive Suppressor Mutants}

Random transposon mutagenesis of strain 3362 (ezrA::tet) was carried out using the mariner transposable element TnYLB-1 as described (Le Breton et al., 2006). Plasmid pMarB was transformed into strain 3362 at $30^{\circ} \mathrm{C}$. Individual colonies carrying the complete transposon plasmid were picked and grown in LB at $37^{\circ} \mathrm{C}$ for $8 \mathrm{~h}$. Aliquots were frozen and stored at $-80^{\circ} \mathrm{C}$. Serial dilutions of each culture were plated on nutrient agar plates containing kanamycin or erythromycin and incubated at $50^{\circ} \mathrm{C}$ overnight to inhibit plasmid replication. The following morning, the clone with the highest ratio of $\mathrm{kan}^{\mathrm{R}} / \mathrm{erm}^{\mathrm{R}}$ colonies on plates was chosen. Appropriate dilutions of the selected clone were plated on nutrient agar plates and incubated at $50^{\circ} \mathrm{C}$ to construct a library of about 70,000 colonies. Cells were scraped off the plates, aliquoted, and frozen. About 75,000 clones of the library were plated on PAB plates supplemented with $10 \mu \mathrm{g}$ $\mathrm{ml}^{-1}$ tetracycline and incubated at $37^{\circ} \mathrm{C}$ for $20 \mathrm{~h}$. Individual colonies were picked and checked for integration of the transposon $\left(\mathrm{kan}^{\mathrm{R}}\right)$, loss of the plasmid $\left(\mathrm{erm}^{\mathrm{S}}\right)$, presence of the ezrA deletion $\left(\right.$ tet $\left.^{\mathrm{R}}\right)$, and checked under the microscope to see loss of the filamentous phenotype when streaked on PAB with tetracycline. Two rounds of backcrosses into strain 3362 were performed to confirm the linkage between transposon insertion and loss of 
tetracycline hypersensitivity. Finally, the site of transposon insertion was determined by performing an inverse PCR amplification on the chromosomal DNA which had been previously digested with TaqI and ligated. Finally, PCR reactions were sequenced and the results aligned with the $B$. subtilis published genome sequence. Oligonucleotides for inverse PCR and sequencing were oIPCR1, -2 and -3 respectively, as described (Le Breton et al., 2006).

\section{Cell Length Measurements}

Cells were grown at $37^{\circ} \mathrm{C}$ in $\mathrm{CM}, \mathrm{LB}, \mathrm{PAB}$, or PAB supplemented with $5 \mathrm{mM} \mathrm{Mg}^{2+}$. At mid-exponential phase for $\mathrm{LB}$ medium or early stationary phase for other media, cells were sampled and stained with Nile Red prior to microscopic examination. At least 100-150 cells were measured in each experiment and all experiments were replicated at least three times. The mean cell length was calculated for each experiment and then averaged over three replicates. Wild type cell length was set as $100 \%$ and relative cell length was calculated for all other strains.

\section{Western Blotting}

For the detection of HA-FtsL shown in Figure 4, cells were grown overnight at $30^{\circ} \mathrm{C}$ in $\mathrm{PAB}$ with $5 \mathrm{mM} \mathrm{MgSO}_{4}, 0.5-2 \%$ xylose, $1 \mathrm{mM}$ IPTG. Cultures were diluted to an O.D.600 of 0.1 in the same medium, grown for $2 \mathrm{~h}$ at $37^{\circ} \mathrm{C}$ and diluted again to 0.1 in warm medium. The exponentially growing cultures were incubated until an O.D.600 of 0.3 (Figure 4A) or 0.5 (Figures 4B,C) was reached. Cell pellets were resuspended in $100 \mu \mathrm{l}$ of $1 \times$ NuPAGE LDS Sample Buffer (Invitrogen) with $5 \times$ Complete mini protease inhibitor (Roche) and broken by sonication. Relative protein concentrations were estimated by reading the A280 of all samples with a NanoDrop $\mathbb{R}$ ND-1000 spectrophotometer and equal amount of proteins were loaded on polyacrylamide gels. Proteins were transferred onto a PVDF membrane (GE Healthcare) by using either a wet or a semi-dry procedure and Western blotting was performed according to standard methods. In this study, a 1:10,000 dilution of rabbit polyclonal anti-FtsZ serum (laboratory stock), 1:10,000 dilution of rabbit polyclonal anti-PBP2B serum (laboratory stock), and a 1:1000 dilution of mouse monoclonal 12CA5 anti-HA antibody (Ivanov and Nasmyth, 2005) were used. Secondary antiserums, anti-rabbithorseradish-peroxidase, and anti-mouse-horseradish-peroxidase (Sigma), were used at a dilution of 1:10,000.

For the immunodetection of ZapA shown in Figure S1, strains were grown at $37^{\circ} \mathrm{C}$ in $\mathrm{PAB}$ medium and samples were collected and flash frozen at O.D.600 0.3. Cell pellets were resuspended in lysis buffer $(100 \mathrm{mM}$ Tris- $\mathrm{Cl} \mathrm{pH} 7.5,2 \mathrm{mM}$ EDTA, supplemented with Roche Complete mini protease inhibitor) containing $5 \mu \mathrm{g} / \mathrm{ml}$ lysozyme, incubated $10 \mathrm{~min}$ at $37^{\circ} \mathrm{C}$ and then sonicated. Cell debris were removed by centrifugation. Relative protein concentrations were estimated with a Bio-Rad protein assay and equal amount of proteins were loaded on NuPAGE 4-12\% Bis-Tris gradient gels which were run in MES buffer (Life Technologies). Proteins were transferred onto a Hybond-P PVDF membrane (GE Healthcare) by using a wet procedure and western blotting was performed according to standard methods. A 1:2000 dilution of rabbit polyclonal anti-ZapA serum was used.
Anti-rabbit horseradish peroxidase-linked antiserum (Sigma) was used as secondary antibody at a dilution of 1:10,000. Protein bands were detected using an ImageQuant LAS 4000 mini digital imaging system (GE Healthcare).

\section{Results}

\section{Tetracycline Hypersensitivity of an ezrA Mutant}

A B. subtilis ezrA mutant forms normal colonies on plate. When we transformed an ezrA deletion into other B. subtilis backgrounds, sometimes very small colonies were obtained that contained filamentous cells. However, this result was not always reproducible. Eventually, it emerged that this filamentous phenotype was caused by insertion of the tetracycline resistance cassette tet $L$ in ezrA, in combination with selection of transformants on selective PAB plates. Without tetracycline, an ezrA mutant forms normal colonies on PAB plates (Figure 1A). Despite the presence of a functional resistance cassette, the addition of tetracycline $(10 \mu \mathrm{g} / \mathrm{ml})$ results in very small colonies containing highly filamentous cells, (Figures 1A,B). Addition of $5 \mathrm{mM} \mathrm{MgSO}_{4}$ to $\mathrm{PAB}$ plates with tetracycline restored normal growth and abolished filamentation (Figures 1A,B). When the tet $L$ cassette was located at another locus (lacA), in an otherwise wild-type background, no effect on cell length or colony size was observed. Subsequent introduction of a different ezrA deletion (ezrA::cat) into this background resulted again in small colonies and strong filamentation on PAB plates with tetracycline (Figures 1A,B). For unknown reasons, we did not observe this filamentation phenotype in liquid PAB medium. Finally, hypersensitivity became apparent also on nutrient agar plates, but only when increased levels of tetracycline were used ( $\geq 30 \mu \mathrm{g} / \mathrm{ml}$ ).

To examine whether the effect of tetracycline was specific for an ezrA mutant, several division mutants were tested that carried a tet $L$ cassette. No significant effect on colony size or cell length was observed when a $z a p A, \operatorname{sepF}, n o c$, or $g p s B$ mutant was grown on $\mathrm{PAB}$ plates with tetracycline (Figure 1A and data not shown), indicating that the effect is specific for ezrA.

\section{Tetracycline Effect is Not Related to Protein Translation Inhibition}

PAB medium contains relative low concentrations of $\mathrm{Mg}^{2+}$ (210 $\mu \mathrm{M}$ Murray et al., 1998). Interestingly, the growth defect of an ezrA mutant on PAB plates with tetracycline can be suppressed by the addition of $\mathrm{Mg}^{2+}$. Tetracycline is a metal-ion chelator (Nelson, 1998) and might reduce the cellular $\mathrm{Mg}^{2+}$ concentrations to such levels that growth and cell division are affected in this growth medium. If this is the case then a similar phenotype should be observed with other magnesium chelators. However, neither the addition of EDTA nor phenanthroline, applied at the same molar concentrations as tetracycline $(23 \mu \mathrm{M})$, had an effect on cell division (not shown).

The tet $L$ cassette encodes the TetA transporter which exports tetracycline in a complex with divalent cations such as $\mathrm{Mg}^{2+}$ (Krulwich et al., 2001). To see whether the growth phenotype was linked to the presence of the TetA transporter, an alternative tetracycline resistance cassette was used. tet- 4 is a point 
A
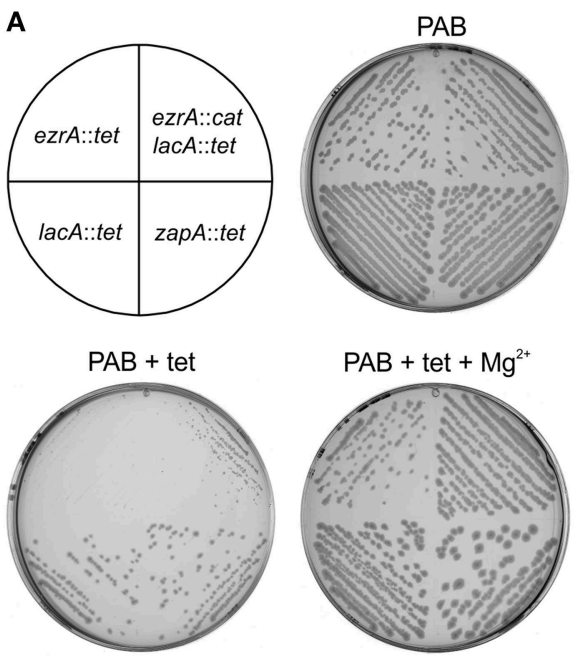

FIGURE 1 | Effect of tetracycline on ezrA mutants. (A) Growth of ezrA::tet (3362), lacA::tet (SG82), ezrA::cat lacA::tet (PG100), and zapA-yshB::tet (1356) strains on PAB plates with and without
B ezrA::cat lacA::tet lacA::tet
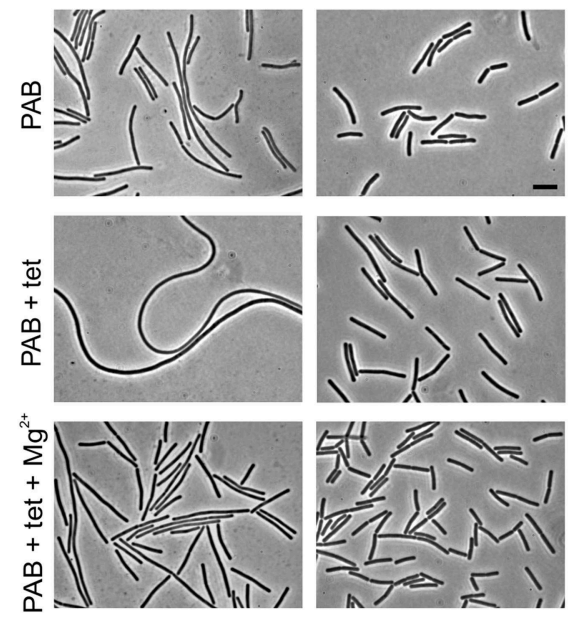

$10 \mu \mathrm{g} / \mathrm{ml}$ tetracycline (tet) and $5 \mathrm{mM} \mathrm{MgSO}_{4}\left(\mathrm{Mg}^{2+}\right)$. (B) Phase contrast images of cells taken from the PAB plates in (A). Scale bar $5 \mu \mathrm{m}$. mutation in the ribosomal protein S10 that reduces the sensitivity of the ribosome for tetracycline (Williams and Smith, 1979; Wei and Bechhofer, 2002). This mutation confers tetracycline resistance without affecting the concentration of the internal $\mathrm{Mg}^{2+}$ pool. The tet- 4 mutation provides a lower resistance to tetracycline compared to the tet $L$ cassette, therefore strains were grown on $\mathrm{PAB}$ plates containing $2 \mu \mathrm{g} / \mathrm{ml}$ tetracycline (Figures 2A,B). Again, introduction of an ezrA mutation in the tet- 4 background caused hypersensitivity to tetracycline, indicating that this phenotype is not related to the TetA transporter.

The fact that the tet-4 mutation is unable to prevent the tetracycline effect suggests that this phenomenon is not associated with the inhibition of protein translation. This was supported by the finding that $0.5 \mu \mathrm{g} / \mathrm{ml}$ anhydrotetracycline, a tetracycline analog that does not bind to the ribosome (Oliva and Chopra, 1992), also affects growth and cell division of ezrA mutants (Figures 2C,D).

\section{Tetracycline Does Not Affect Z-Ring Formation}

The tetracycline-induced filamentation of ezrA cells indicates a cell division problem. To examine whether this problem is caused by an inability to form Z-rings, a fluorescent GFP-FtsZ marker was introduced. The resulting strain PG209 (ezrA::tet amyE::Pxyl-gfp-ftsZ) was streaked on PAB plates containing $10 \mu \mathrm{g} / \mathrm{ml}$ tetracycline and $0.5 \%$ xylose to induce GFP-FtsZ. Cells were taken from colonies and mounted onto agarose covered microscope slides containing Nile Red and DAPI, to stain the cell membrane and nucleoid, respectively (Figure 3 ). The filamentous cells were very fragile and many cells lysed. Intact cells showed normal nucleoids and some Z-rings, but the fluorescent membrane stain indicated a clear lack of septation. This suggests that the block in cell division is not caused by a defect in FtsZ assembly, but occurs later in the division process.

\section{Low FtsL Levels in ezrA Mutants}

Kawai and Ogasawara have shown that an ezrA mutant is sensitive to reduced FtsL expression levels (Kawai and Ogasawara, 2006). FtsL is unstable and cleaved by the zinc metalloprotease RasP, which is involved in regulated intramembrane proteolysis (RIP) (Heinrich et al., 2008; Wadenpohl and Bramkamp, 2010). This proteolytic degradation plays an important regulatory role in the assembly of the late cell division proteins (Daniel et al., 2006). Possibly, FtsL levels become critically limiting for growth when an ezrA mutant is grown on PAB plates in the presence of tetracycline.

We first tested FtsL levels in an ezrA background, by using a strain that carries a deletion of the native $f t s L$ gene and an ectopically located xylose-inducible HA-tagged ftsL fusion (strain 814, Daniel and Errington, 2000). The HA epitope tag enables convenient detection of cellular FtsL levels with Western blotting using sensitive HA-antibodies. Strain 814 was transformed with the ezrA::tet mutation, resulting in strain PG305. This strain showed an extremely slow growth rate when grown with $0.5 \%$ xylose in liquid PAB medium without antibiotics. In fact, no HA-FtsL could be detected under these conditions (Figure 4A). When the xylose concentration was increased to $2 \%$, strain PG305 grew better, although still slower than strain 814, and a weak HAFtsL band became visible (Figure 4B). Interestingly, strain PG305 showed normal growth with $0.5 \%$ xylose when $5 \mathrm{mM} \mathrm{Mg}^{2+}$ was added to the medium, although FtsL levels were still not restored to the levels observed in the parental strain 814 (Figure 4C). These data indicate that a deletion of ezrA results in reduced FtsL levels, which explains why an ezrA mutant is so sensitive for manipulation of the FtsL concentration.

As mentioned above, we observed hypersensitivity to tetracycline only on agar plates. Unfortunately, growth on solid medium hampers homogeneous sampling at specific growth phases. Therefore, we introduced a tetracycline resistance marker 
A

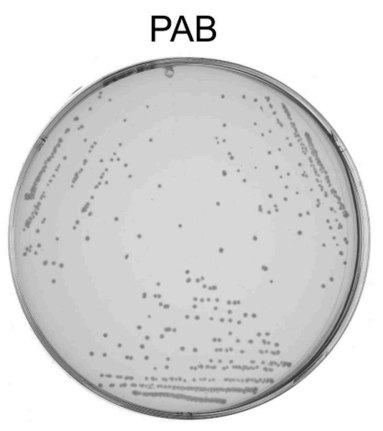

B

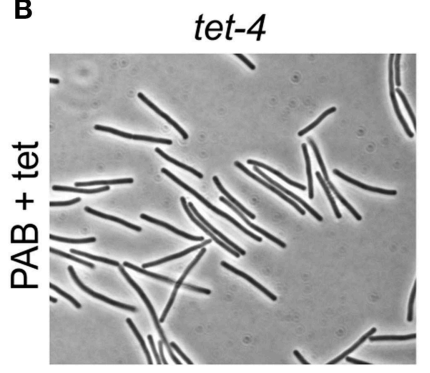

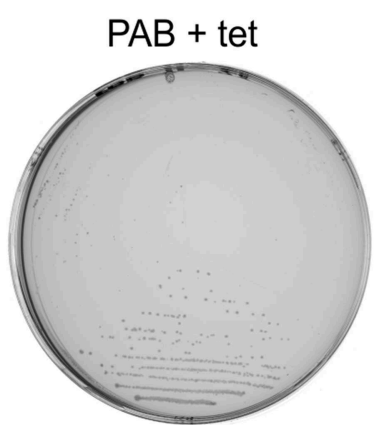

C

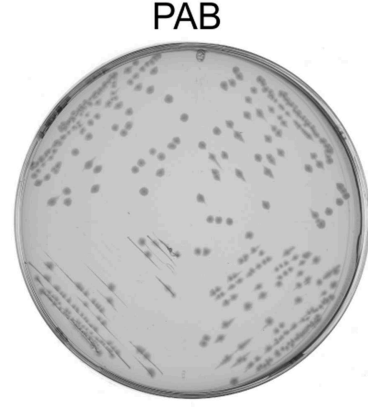

D

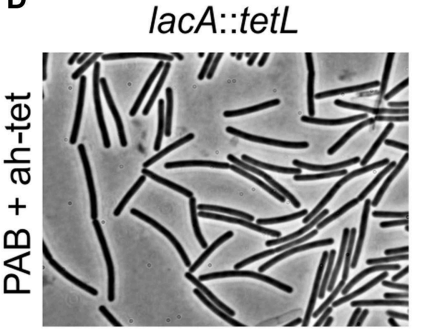

ezrA::spc tet-4

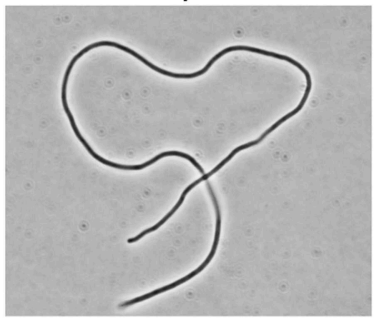

$\mathrm{PAB}+$ ah-tet
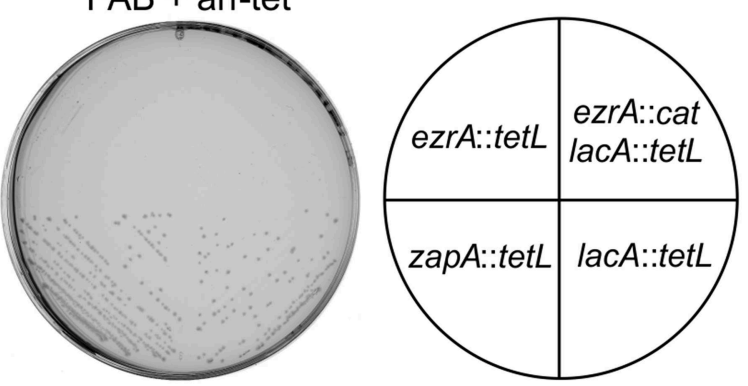

ezrA::tetL

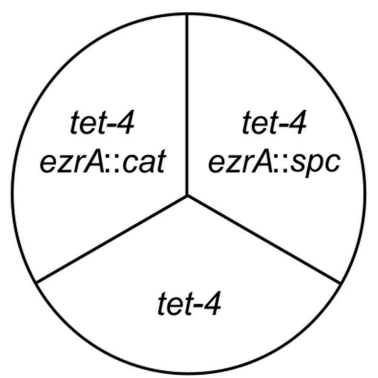

ezrA::cat tet-4
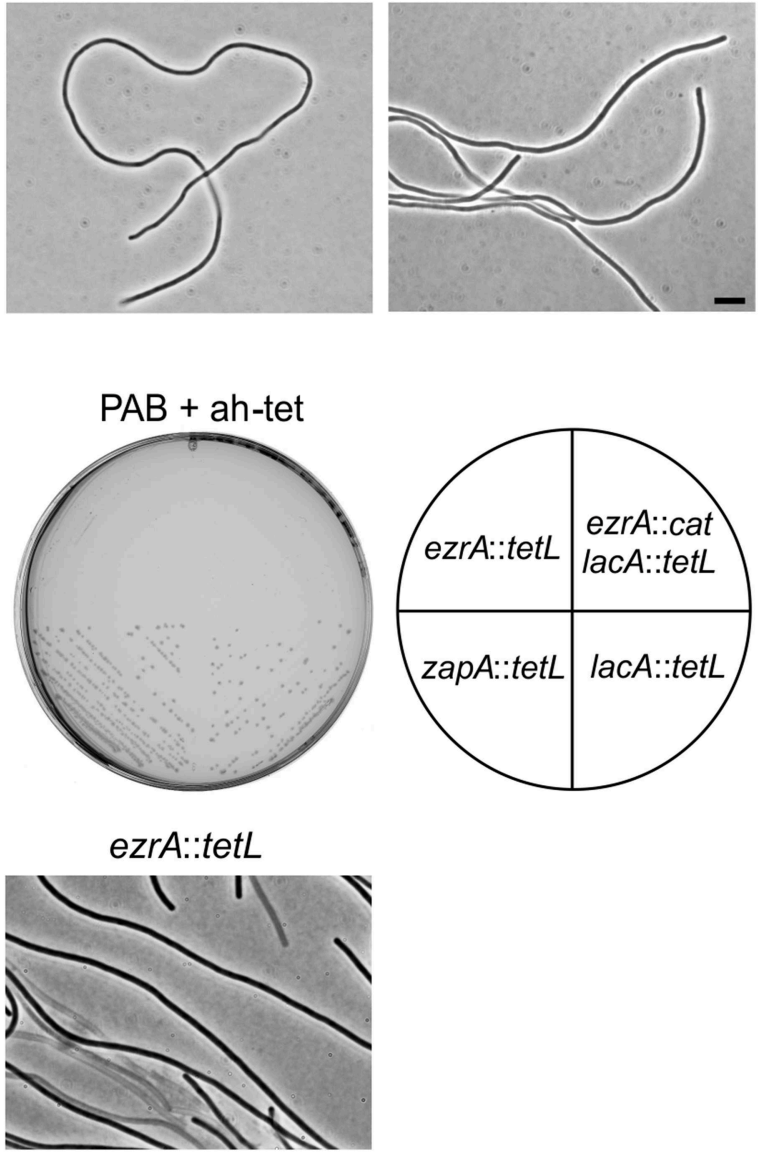

FIGURE 2 | Tetracycline-induced growth defects of ezrA mutants are unrelated to protein translation inhibition. (A) Growth of strains tet-4 (PG112), ezrA::cat tet-4 (PG116), ezrA::spc tet-4 (PG113), on PAB plates supplemented with $2 \mu \mathrm{g} / \mathrm{ml}$ tetracycline (tet). (B) Phase contrast images of cells taken from the PAB plates in (A). Scale bar $5 \mu \mathrm{m}$. (C) Effect of anhydrotetracycline. Growth of ezrA::tet (3362), ezrA::cat lacA::tet (PG100), zapA-yshB::tet (1356), and lacA::tet (SG82) strains on PAB plates with or without $0.5 \mu \mathrm{g} / \mathrm{ml}$ anhydrotetracycline (ah-tet). ZapA mutant strain was included as an additional control. (D) Phase contrast images of cells taken from the PAB plates in (C). into strain 814, obtaining strain PG742 and plated serial dilutions onto $\mathrm{PAB}$ plates in the presence of increasing concentrations of xylose, to allow for differential expression of FtsL. As shown in Figure 4D, higher levels of induction were required to allow colony formation in the presence of tetracycline, suggesting that FtsL levels become limiting under those conditions. These data would imply that the tetracycline-induced filamentation of an ezrA mutant can be suppressed by increasing FtsL levels in the cell. To test this, a xylose-inducible truncated copy of FtsL $(\Delta 30-f t s L)$ was introduced into an ezrA::tet mutant. This variant of FtsL was chosen since removal of the first 30 amino acids of FtsL stabilizes the protein (Bramkamp et al., 2006). When the resulting strain PG160 was streaked on $\mathrm{PAB}$ plates with tetracycline and $1 \%$ xylose, cell 


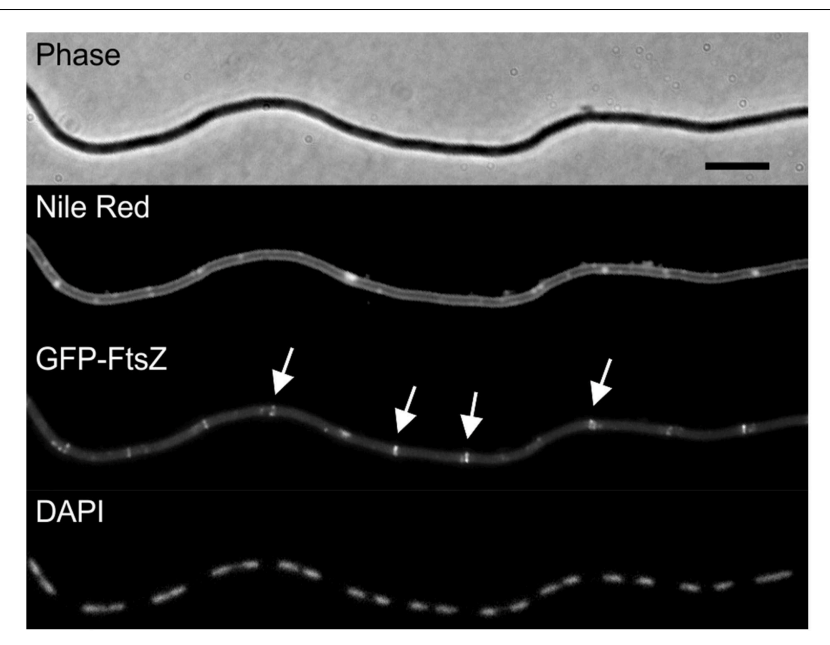

FIGURE 3 | Tetracycline does not prevent Z-ring formation. Strain PG209 (ezrA::tet amyE::Pxyl-gfp-ftsZ) was streaked on PAB plates containing $10 \mu \mathrm{g} / \mathrm{ml}$ tetracycline, and $0.5 \%$ xylose to induce GFP-FtsZ. Cells were stained with DAPI and Nile Red to visualize nucleoids and the cell membrane, respectively. Arrows highlight some of the Z-rings. Scale bar $5 \mu \mathrm{m}$.

division was indeed restored and normal colonies were obtained (Figures 4E,F).

\section{Screen for Novel Suppressor Mutants}

The mechanism by which tetracycline causes filamentation of an ezrA mutant is unclear. To examine whether other proteins are involved in the tetracycline effect, we screened a transposon library for mutants that would grow normally on PAB plates with tetracycline. Plasmid pMarB, carrying the mariner transposon TnYLB-1 (Le Breton et al., 2006), was introduced into strain 3362 (ezrA::tet), and after transposon mutagenesis approximately 70,000 colonies were screened. Several suppressor mutants that restored colony growth and rescued the division defect were selected. Further analyses showed that one suppressor strain contained a transposon inserted immediately after $z a p A$. Two other suppressor mutants harbored transposon insertions into galE, which encodes an UDP-galactose epimerase (Estrela et al., 1991; Soldo et al., 2003), and two suppressor mutants contained transposon insertions in the unknown gene $y p m B$. A galE and $y p m B$ null mutant were made by replacing the complete ORFs with a kanamycin resistance marker (strain PG234 and PG235), and transforming the deletions into strain 3362 (ezrA::tet). The resulting double mutants grow normally on PAB plates with tetracycline (Figure 5), confirming that the absence of either GalE or YpmB suppresses the tetracycline induced cell division defect.

\section{ZapA Overexpression Suppresses Filamentation}

$z a p A$ is the upstream gene in the bicistronic $z a p A-y s h B$ operon. Since one of the suppressors contained a transposon insertion between $z a p A$ and $y s h B$, and precisely one nucleotide upstream the start codon of $y s h B$, it is possible that a reduced expression of YshB prevents the synthetic filamentous phenotype. To test this, an ezrA mutation was introduced into a strain that lacks the complete $z a p A-y \operatorname{sh} B$ operon and contains an ectopic copy of only zapA driven by the IPTG-inducible Pspac promoter. In the absence of IPTG, this strain formed small colonies and highly filamentous cells on PAB plates, even without tetracycline (Figures 6A,B). This is in agreement with a previous study which showed that a $z a p A$ ezrA double mutant forms very filamentous cells (Gueiros-Filho and Losick, 2002). However, the strain grew normally and showed no filamentation when IPTG was added to induce ZapA expression (Figures 6A,B), even in the presence of tetracycline (not shown). Thus, suppression of the tetracycline phenotype does not require the absence of YshB, since an ezrA mutant that overexpresses ZapA and that contains a normal copy of the $z a p A-y s h B$ operon, also grows normally (Figure 5). It is likely that the transposon insertion somehow stabilizes $z a p A$ mRNA leading to increased ZapA levels in the cell. We therefore tested ZapA levels with Western blot and confirmed that the transposon insertion causes overexpression of ZapA in both 168 and ezrA backgrounds (Figure S1).

\section{Deletion of galE Restores Cell Division}

GalE is an epimerase that catalyzes the reversible conversion between UDP-galactose and UDP-glucose, as well as between UDP-N-acetylgalactosamine and UDP-N-acetylglucosamine (Krispin and Allmansberger, 1998; Soldo et al., 2003). A galE mutant is defective in exopolysaccharide synthesis which results in impaired biofilm formation (Chai et al., 2012). Moreover, the cell wall of a galE mutant is devoid of poly(glucose galactosamine 1-P), the so-called minor wall-teichoic acid (Freymond et al., 2006). Teichoic acids are phosphate-rich anionic glycopolymers which constitute a major component of the Gram-positive cell wall. Several physiological roles have been proposed for these polymers, including cation homoeostasis, antibiotic resistance, morphogenesis and cell division (Weidenmaier and Peschel, 2008; Schirner et al., 2009). Interestingly, an ezrA mutant is also more sensitive to chloramphenicol and ampicillin as well as to several other cell wall antibiotics (Figures S2, S3). General antibiotic sensitivity is a phenotype that is often observed in cell wall mutants, and in this case might be related to the role of EzrA in shuttling PBP 1 from the lateral wall to the division site (Claessen et al., 2008). Introduction of a galE mutation decreased antibiotic sensitivity of an ezrA mutant to wild type levels (Figure S3). Importantly, inactivation of galE alone showed no increased resistance to antibiotics compared to the wild type strain 168, suggesting that the suppression effect is not due to a general increased protection against antibiotics by means of an altered cell wall composition. Moreover, deletion of the sugar transferases GgaAB, which also impairs minor teichoic acid synthesis (Freymond et al., 2006), did not suppress the tetracycline phenotype.

\section{Absence of GalE Restores Cell Division in sepF ezrA and zapA ezrA Double Mutants}

A remaining question is whether the absence of GalE only suppresses the tetracycline effect or whether such mutation actually has a more direct role in cell division. Previously, it was shown that mutations in the lipoteichoic acid biosynthesis pathway reduces the activity of UgtP, thereby stimulating FtsZ polymerization (Weart et al., 2007). Since a galE mutation changes 


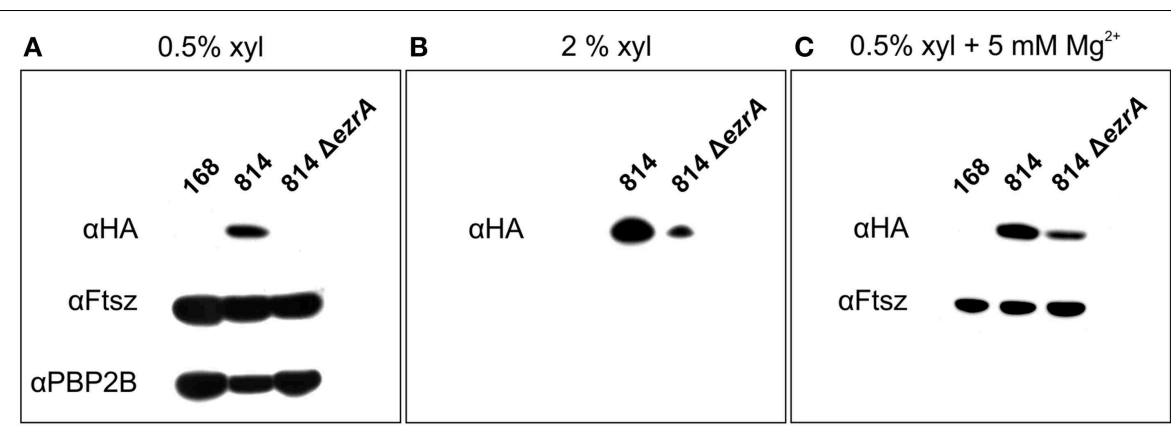

D - Tet + Tet

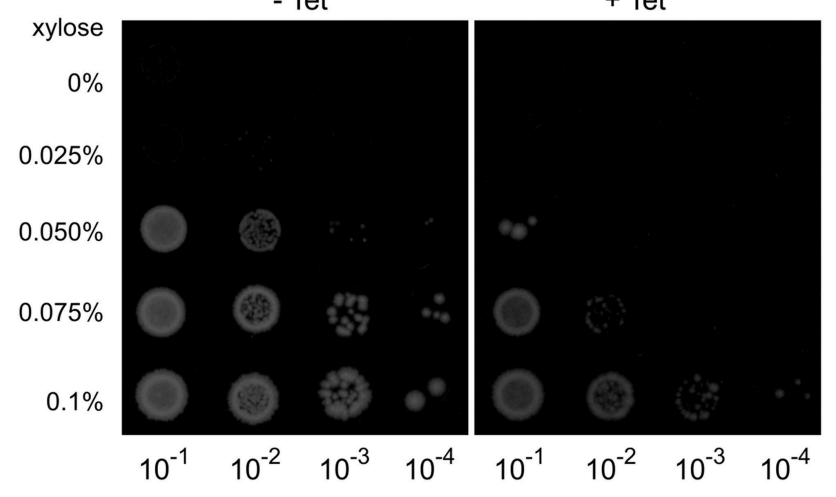

E
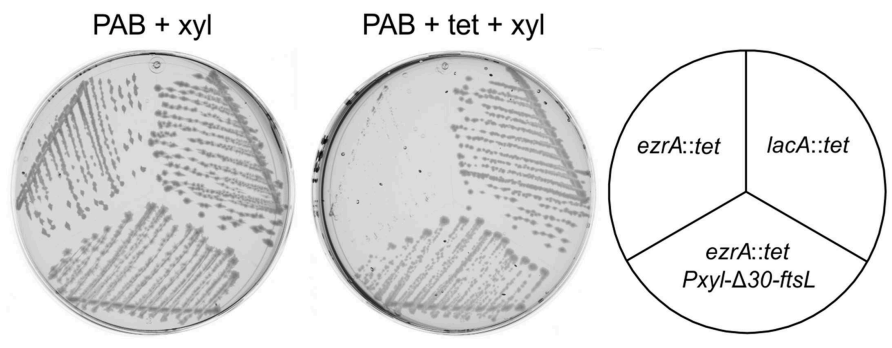

$\mathbf{F}$
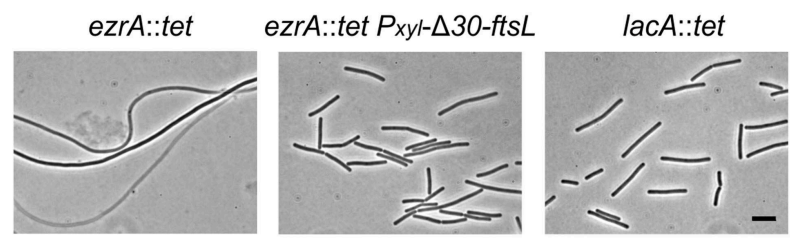

FIGURE 4 | FtsL overexpression suppresses the tetracycline effect. (A) Western blot of HA-FtsL, FtsZ, and Pbp2B from total protein extracts of strains 168 (wild type), 814 ( $\Delta$ ftsL-Pspac-pbpB, amyE::Pxyl-HA-ftsL), and $814 \Delta$ ezrA (PG305) grown at $37^{\circ} \mathrm{C}$ in PAB medium supplemented with $1 \mathrm{mM}$ IPTG and $0.5 \%$ xylose. IPTG was added to express the essential pbpB gene downstream of the ftsl-pbp2B operon. (B) Western blot of HA-FtsL from total protein extracts of strains 814 and $814 \triangle$ ezrA (PG305) grown in PAB medium with $1 \mathrm{mM}$ IPTG and $2 \%$ xylose. (C) Western blot of HA-FtsL and FtsZ from total protein extracts of strains 168,814 , and $814 \Delta$ ezrA (PG305) grown at $37^{\circ} \mathrm{C}$ in PAB medium supplemented with $5 \mathrm{mM} \mathrm{MgSO}_{4}, 20 \mu \mathrm{g} / \mathrm{ml} \mathrm{K}$-aspartate, $1 \mathrm{mM}$ IPTG, and
$0.5 \%$ xylose. Aspartate was included to circumvent any effect on the inactive downstream aspB gene. (D) Growth of strain PG742

( $\Delta$ ftsL-Pspac-pbpB, amyE::Pxyl-HA-ftsL, lacA::tet) on PAB plates supplemented with $1 \mathrm{mM} \mathrm{IPTG}$, with increasing concentrations of xylose $(0.025-0.1 \%)$ and with or without $10 \mu \mathrm{g} / \mathrm{ml}$ tetracycline. Serial dilutions of exponentially growing cells were plated and images were taken after overnight incubation at $37^{\circ} \mathrm{C}$. (E) Growth of ezrA::tet (3362), lacA::tet (SG82), and ezrA::tet amyE::Pxyl- $\triangle 30$-ftsL (PG160) strains on PAB plates with $1 \%$ xylose, and with or without $10 \mu \mathrm{g} / \mathrm{ml}$ tetracycline, after overnight incubation at $37^{\circ} \mathrm{C}$. (F) Phase contrast images of cells taken from the plates. Scale bar $5 \mu \mathrm{m}$. the teichoic acid composition of the cell, this mutation might also influence cell division. To test this, the possible mitigating effect of a galE deletion on the cell division defect of a $z a p A$ ezrA double mutant was investigated. A strain lacking ezrA, and with $z a p A$ under control of an IPTG-inducible promoter (strain PG164), forms small colonies and filamentous cells in 

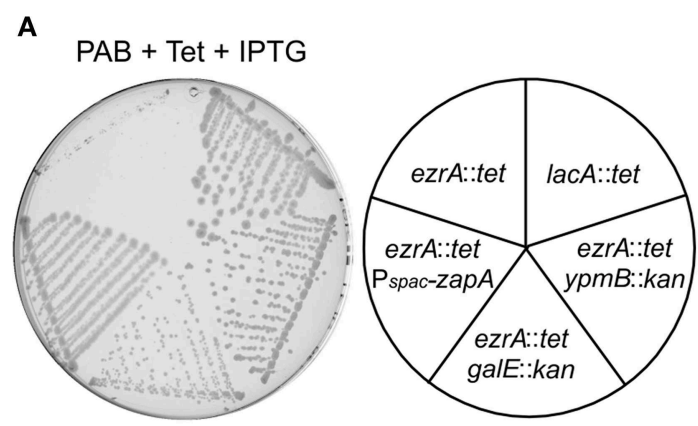

B
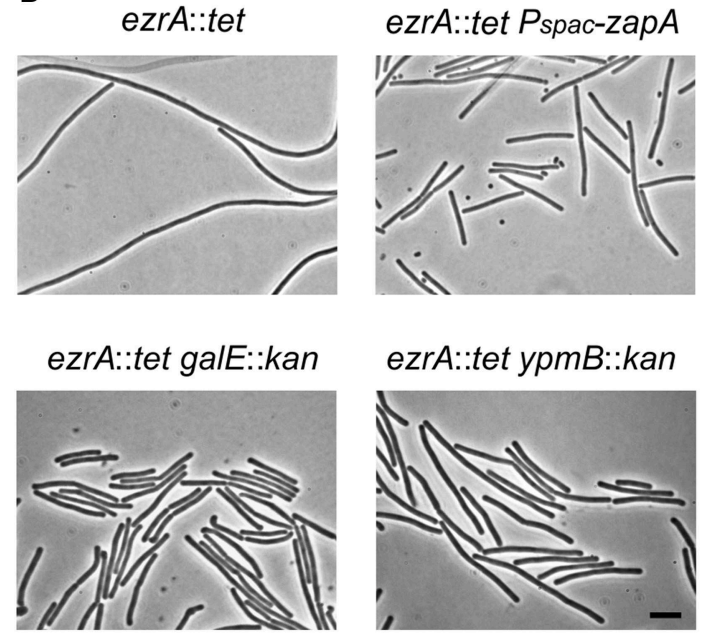

FIGURE 5 | Suppression of the tetracycline phenotype. (A) Growth of ezrA::tet (3362), ezrA::tet aprE::Pspac-zapA (PG273) ezrA::tet galE::kan (PG238), ezrA::tet ypmB::kan (PG239), lacA::tet (SG82) strains on a PAB plate with $10 \mu \mathrm{g} / \mathrm{ml}$ tetracycline and $1 \mathrm{mM}$ IPTG for ZapA induction. (B) Phase contrast images of cells taken from the PAB plates in (A). Scale bar $5 \mu \mathrm{m}$.

the absence of IPTG. When the galE deletion was introduced into this background, the resulting strain (PG307) grew much better without IPTG and filamentation was strongly reduced (Figures 6A,B).

Since a galE deletion restored cell division in the zapA ezrA double mutant, we were curious whether such deletion could also restore growth and cell division in the synthetic lethal sepF ezrA double mutant. B. subtilis strain 4077 contains an ezrA deletion and an IPTG-inducible sepF operon. This strain can only grow when IPTG is added to the growth medium (Hamoen et al., 2006). However, transformation of the galE mutation into this strain resulted in colony formation on PAB plates without IPTG (Figure 6C), and microscopic imaging showed that cell division was restored (Figure 6D). Consistent with this result, it was possible to make a viable ezrA sepF galE triple mutant (PG294), although this strain grows slower than the single mutants and shows a high degree of filamentation (Figure S4). Again, the effect of a galE deletion is not linked to the lack of minor teichoic acids as the $\triangle g g a A B$ mutant failed to suppress IPTG dependency of strain 4077 (not shown). Therefore, we must conclude that GalE activity affects the cell division process.

\section{Deletion of ypmB (tseB) Suppresses the Tetracycline Effect}

Two transposon suppressors were found in $y p m B$, and replacement of $y p m B$ by a kanamycin resistant marker suppressed the tetracycline-induced growth inhibition and filamentation (Figure 5). However, $y p m B$ is the second gene of a tri-cistronic operon and is preceded by $y p m A$ and followed by aspB, which is involved in aspartate biosynthesis. To rule out a possible downstream effect, an ectopic IPTG-inducible copy of $y \mathrm{pmB}$ was introduced into the $y p m B$ ezrA double mutant. The resulting strain PG333 forms only filamentous cells on tetracycline containing PAB plates when IPTG is present, indicating that the transposon suppression is due to the absence of a functional $y p m B$ gene and not a consequence of downstream effects on $a s p B$ (Figure S5). Because of its role in the tetracycline sensitivity of an ezrA strain, this hypothetical gene was renamed $t s e B$ (tetracycline sensitivity suppressor of $\underline{e} z r A$ ).

When cell lengths of the different transposon mutants were measured in an otherwise wild type background, the insertion in $t s e B$ showed the greatest effect and produced significant shorter cells compared to the wild type strain, especially when grown in minimal competence medium (approximately 25\% shorter) (Figures 7A,B). Minimal competence medium contains a relative high concentration of $\mathrm{Mg}^{2+}(6.6 \mathrm{mM})$, and the addition of magnesium to $\mathrm{PAB}$ medium further reduced the average cell length (Figure 7B). The addition of aspartic acid to the growth medium, which might be required if $a s p B$ was not expressed at sufficient levels, did not have an effect on this phenotype (not shown).

Secondary structure predictions of the 161 amino acid long TseB suggested that the protein has a large extracellular domain attached to the cell membrane by a single $\mathrm{N}$-terminal transmembrane helix (SOSUI software, Hirokawa et al., 1998). To study the localization of TseB, an N-terminal GFP-TseB fusion was constructed using a monomeric variant of GFP. The reporter fusion was inserted into the amyE locus of a strain carrying also the wild type copy of $t s e B$ at the native locus. The GFP-TseB fusion is at least partially functional since it can complement the short cell phenotype of a $t s e B$ mutation in minimal medium (not shown). The GFP-TseB fusion shows clear membrane localization that is enriched at cell division sites in some cells (Figure 7C). In addition, the GFP signal appears to be almost absent from matured septa (Figure 7C, arrows). Time-lapse microscopy showed this dynamic localization more clearly, and confirmed the disappearance of the protein from septa late in the cell division process, presumably when septation is completed (Figure 7D).

\section{Discussion}

\section{Hypersensitivity to Tetracycline}

The finding that a cell division mutant is hypersensitive to antibiotics, and in particular to tetracycline, has not been reported before. It is as yet unclear why tetracycline causes a growth and division defect in an ezrA mutant while the tetracyclineresistance marker is present. Our data suggests that FtsL might be destabilized under these growth conditions (Figure 4D). This might lead to a severe division block when combined with an 


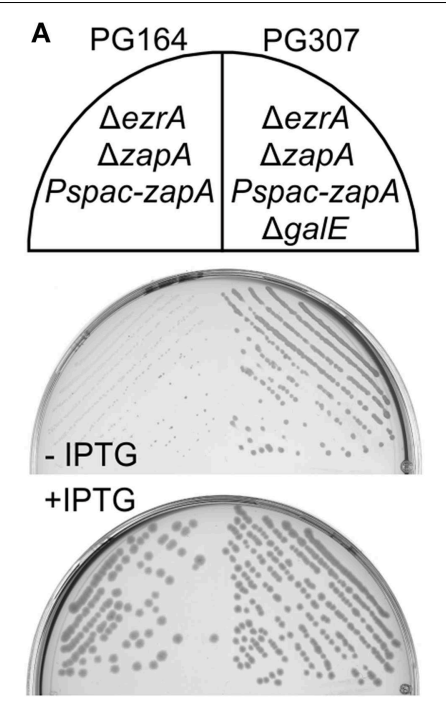

B
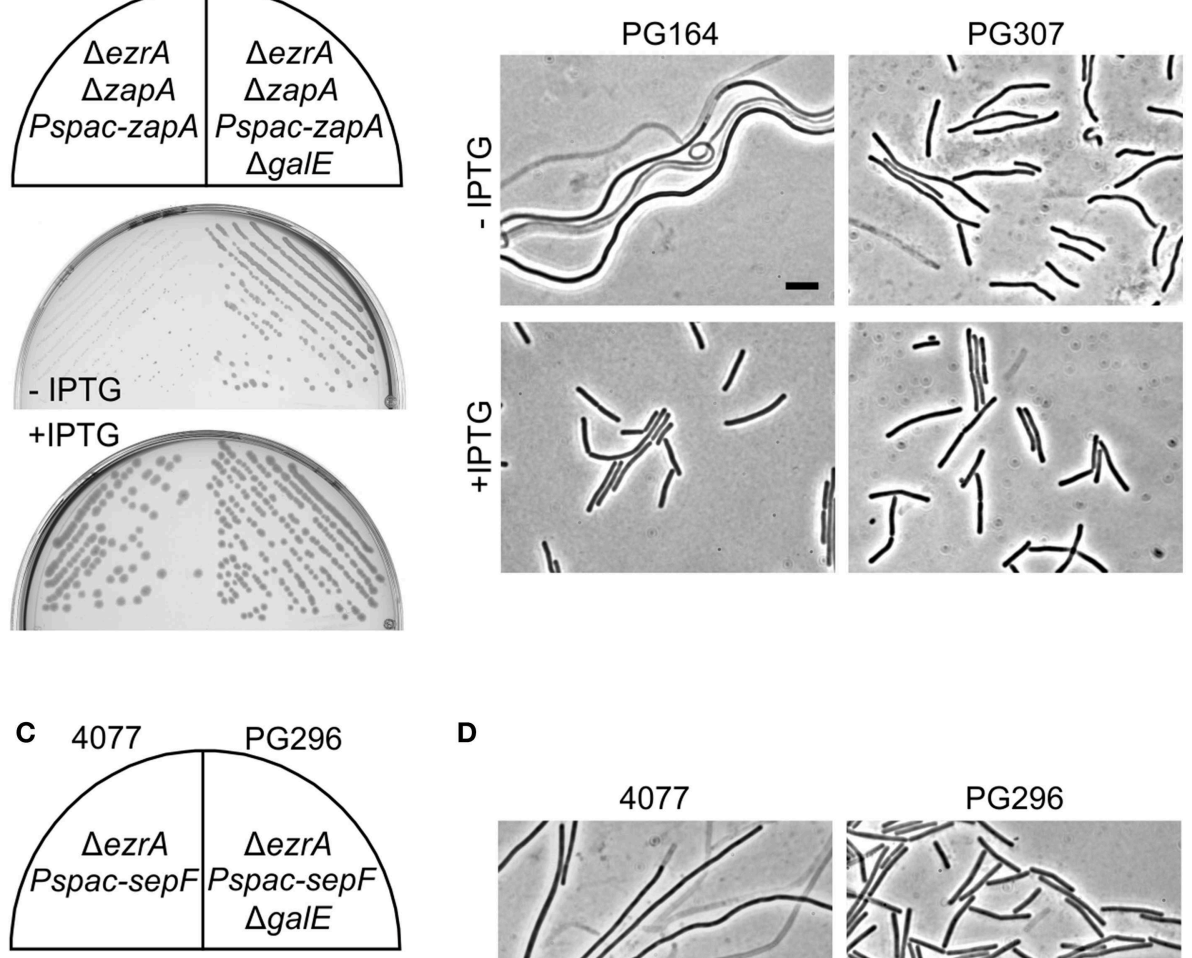

D

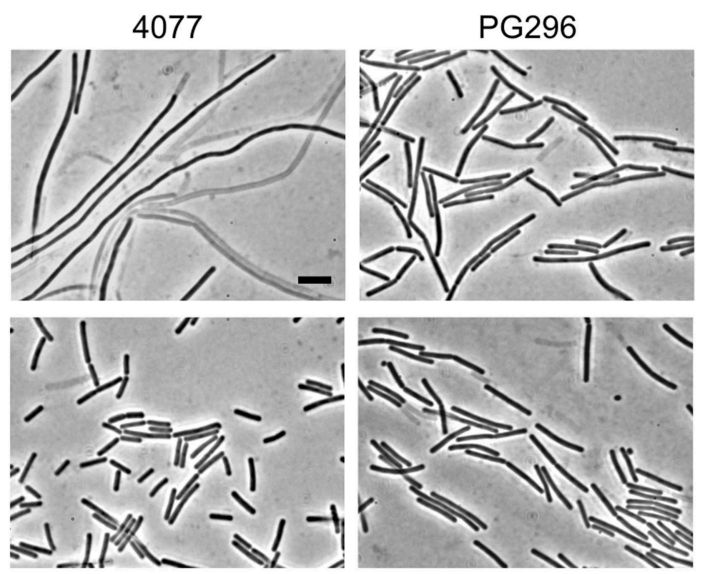

FIGURE 6 | Absence of GalE restores cell division in zapA ezrA and sepF ezrA double mutants. (A) Growth on PAB plates with or without $1 \mathrm{mM}$ IPTG, and (B) related phase contrast microscopic images of strains PG164 (zapA-yshB::tet, ezrA::cat, aprE::Pspac-zapA) and PG307 (zapA-yshB::tet, ezrA::cat, aprE::P spac-ZapA, galE::kan). IPTG was used to induce ZapA. (C)
Growth on nutrient agar plates with $0.5 \mu \mathrm{g} / \mathrm{ml}$ erythromycin, in the presence or absence of $1 \mathrm{mM} \mathrm{IPTG}$, and (D) related phase contrast microscopic images of strains 4077 (y/mBC::Pspac-y/mD-H, ezrA::tet) and PG296 (ylmBC:: $P_{\text {spac }}-y / m D-H$, ezrA::tet, galE::kan). Addition of IPTG induces the expression of $\operatorname{sep} F(=y / \mathrm{mF})$ and of the $y / m D E G H$ genes. Scale bar $5 \mu \mathrm{m}$.
ezrA deletion, which has in itself a similar effect (Figures 4A-C). However, our transposon screen revealed that also ZapA overexpression can suppress the tetracycline effect. In contrast to FtsL, ZapA is an early cell division protein and forms links between FtsZ protofilaments promoting Z-ring assembly (Gueiros-Filho and Losick, 2002; Pacheco-Gomez et al., 2013). Possibly, this also promotes the stability of the late divisome components.

The fact the tetracycline hypersensitivity phenotype is only observed on PAB plates and not in liquid medium might have to do with localized depletion of $\mathrm{Mg}^{2+}$ ions that exacerbates the effect. Since $\mathrm{Mg}^{2+}$ suppresses the tetracycline induced phenotype, we initially assumed that the metal-ion chelating activity of tetracycline was responsible for the cell division defect. However, other metal chelators did not result in filamentation of an ezrA mutant. Interestingly, it is not the classical translationinhibiting activity of tetracycline that is causing cell filamentation, since the tet-4 ribosomal mutation did not mitigate the tetracycline effect, and anhydrotetracycline also caused filamentation. Tetracycline and anhydrotetracycline are lipophilic compounds that accumulate in the cell membrane, and it has been suggested that the bactericidal activity of anhydrotetracycline is caused by membrane de-energization (Chopra, 1994; Chopra and Roberts, 2001). Reduction of the membrane potential by tetracycline could explain its effects on cell division, since the cell 


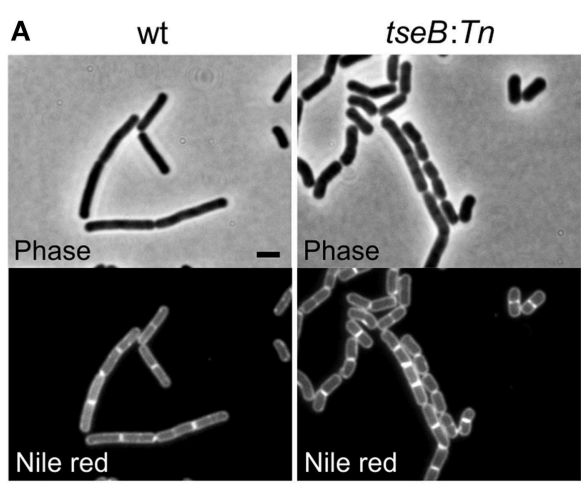

\begin{tabular}{|c|c|c|c|c|}
\hline & CM & LB & PAB & $\mathrm{PAB}+\mathrm{Mg}^{2+}$ \\
\hline 168 & $\begin{array}{c}2.6 \mu \mathrm{m} \\
100 \%( \pm 3)\end{array}$ & $\begin{array}{c}4.5 \mu \mathrm{m} \\
100 \%( \pm 4)\end{array}$ & $\begin{array}{c}6.0 \mu \mathrm{m} \\
100 \%( \pm 12)\end{array}$ & $\begin{array}{c}3.2 \mu \mathrm{m} \\
100 \%( \pm 5)\end{array}$ \\
\hline tseB:Tn & $\begin{array}{c}2.0 \mu \mathrm{m} \\
74 \%( \pm 3)\end{array}$ & $\begin{array}{c}4.1 \mu \mathrm{m} \\
91 \%( \pm 4)\end{array}$ & $\begin{array}{c}5.7 \mu \mathrm{m} \\
96 \%( \pm 13)\end{array}$ & $\begin{array}{c}2.6 \mu \mathrm{m} \\
82 \%( \pm 3)\end{array}$ \\
\hline galE:Tn & $\begin{array}{c}2.5 \mu \mathrm{m} \\
95 \%( \pm 5)\end{array}$ & $\begin{array}{c}4.6 \mu \mathrm{m} \\
101 \%( \pm 3)\end{array}$ & $\begin{array}{c}6.3 \mu \mathrm{m} \\
105 \%( \pm 5)\end{array}$ & $\begin{array}{c}3.0 \mu \mathrm{m} \\
94 \%( \pm 2)\end{array}$ \\
\hline zapA-Tn & $\begin{array}{c}2.5 \mu \mathrm{m} \\
95 \%( \pm 3)\end{array}$ & $\begin{array}{c}4.5 \mu \mathrm{m} \\
99 \%( \pm 4)\end{array}$ & $\begin{array}{c}5.6 \mu \mathrm{m} \\
94 \%( \pm 8)\end{array}$ & $\begin{array}{c}3.0 \mu \mathrm{m} \\
95 \%( \pm 6)\end{array}$ \\
\hline
\end{tabular}
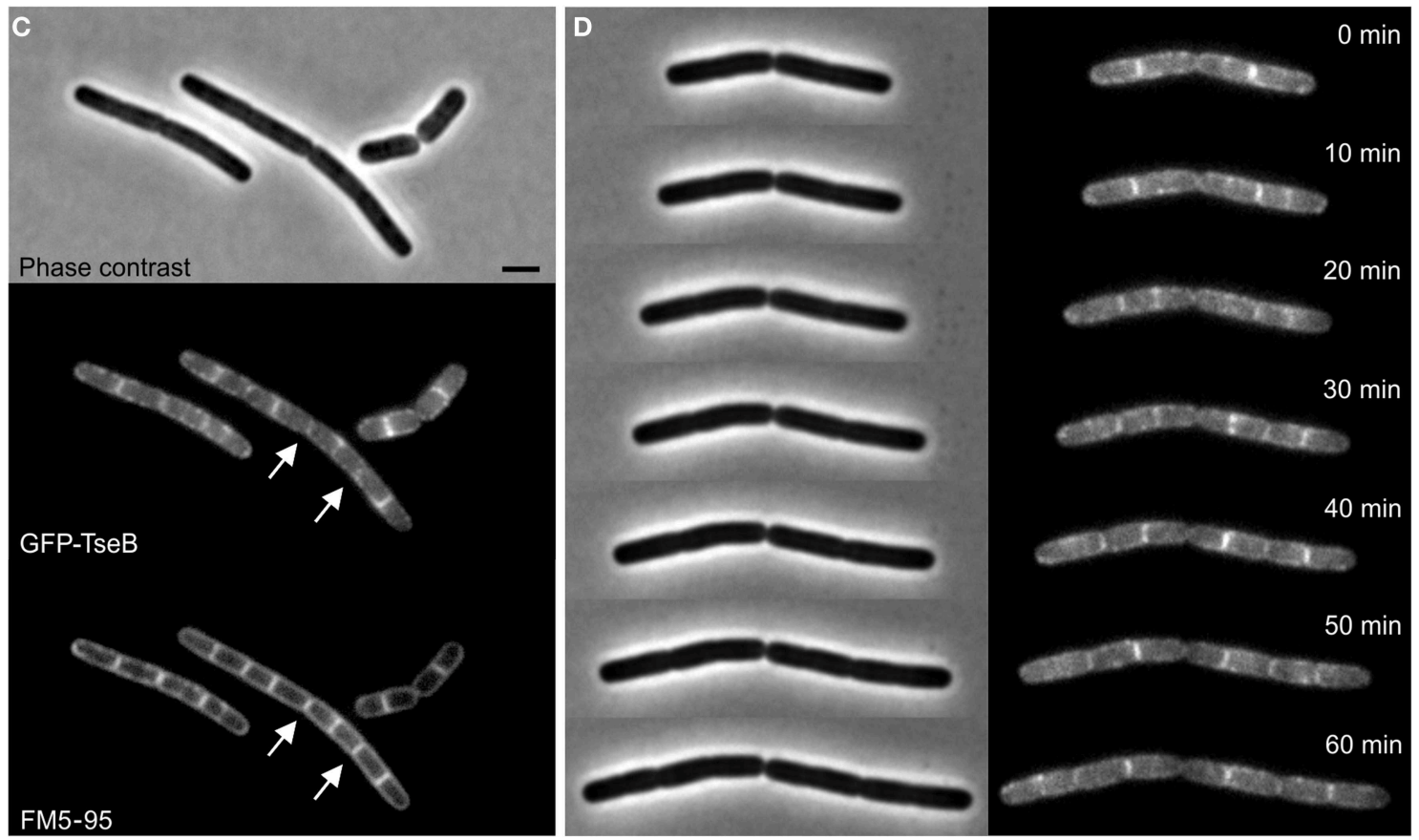

FIGURE 7 | Phenotype of $\Delta$ tseB and localization of GFP-TseB. (A) Phase contrast and membrane stain (Nile red) images of wild type strain 168 and the tse $B$ mutant strain PG135 (tseB:TnYLB-1) grown in competence medium at $37^{\circ} \mathrm{C}$. Scale bar $2 \mu \mathrm{m}$. (B) Cell length measurements of the transposon mutants in different growth media. Strains 168, tseB:TnYLB-1(PG135), galE:TnYLB-1 (PG143) and zapA-TnYLB1-yshB (PG140), were grown at $37^{\circ} \mathrm{C}$ in competence medium (CM), LB, PAB, or PAB supplemented with $5 \mathrm{mM} \mathrm{Mg}^{2+}$ Averaged absolute and relative cell lengths are presented below in \%, and standard deviations are shown in brackets. One hundred to one hundred and fifty cells were measured in each experiment in triplicate. (C) Localization of GFP-TseB. Strain PG718 (amyE::Pxyl-mgfp-tseB) was grown in competence medium at $30^{\circ} \mathrm{C}$ with $0.5 \%$ xylose to express GFP-TseB. GFP, membrane stain (FM5-95) and phase contrast images were taken during exponential growth. Scale bar $2 \mu \mathrm{m}$. Arrows highlight some of the septa in which the GFP signal is absent. (D) Time-lapse microscopy experiment showing dynamic localization of GFP-TseB. Strain PG718 (amyE::Pxyl-mgfp-tseB) was grown at $30^{\circ} \mathrm{C}$ on a microscope slide made of competence medium supplemented with $0.5 \%$ xylose. GFP and phase contrast images were taken every $10 \mathrm{~min}$. division proteins FtsA and MinD require the membrane potential for membrane localization and function (Strahl and Hamoen, 2010). However, we have been unable to detect a clear reduction in membrane potential (within $15 \mathrm{~min}$ ) when $B$. subtilis cells were incubated with tetracycline. It has been shown that relative small amounts of tetracycline $(1 \mu \mathrm{g} / \mathrm{ml})$ can increase the membrane fluidity (Vincent et al., 2004). Possibly, this change in membrane fluidity will make certain transmembrane proteins more susceptible to proteolytic degradation, such as FtsL or other late division proteins. Interestingly, divalent cations are known to reduce membrane fluidity (Binder and Zschornig, 2002; Vest et al., 2004), and this might explain why the addition of $\mathrm{Mg}^{2+}$ suppresses the tetracycline effect.

\section{Effect of GalE on Cell Division}

A galE deletion suppresses the tetracycline effect and rescues the synthetic lethality of $z a p A$ ezrA and sepF ezrA double mutations. This, together with the fact that the lack of minor teichoic acids itself ( $\triangle g g a A B$ mutant) did not suppress the cell division effect, suggests that GalE plays a more direct role in cell division. We 
could not observe any division defect in a galE mutant, but the galE mutation improves the efficiency of division in ezrA mutant cells considerably. One possibility is that the absence of $\mathrm{galE}$ alters the levels of UDP-glucose, since GalE catalyzes the reversible production of UDP-glucose from UDP-galactose. UDP-glucose is the substrate for UgtP, the sugar transferase that is involved in lipoteichoic acid, which also regulates FtsZ assembly (Weart et al., 2007). Therefore, a galE deletion might indirectly influence the activity of UgtP. Interestingly, we were unable to test the ugtP mutant on PAB plates, since this strain showed strongly impaired growth and morphological defects (bulging) in PAB medium (not shown).

\section{TseB Influences Cell Division}

We have shown that TseB deletion suppresses the tetracycline effect and causes a short cell phenotype under certain growth conditions. The protein is attached to the membrane and contains an extra-cytoplasmic peptidase domain that is found in cellwall associated regulatory metallopeptidases (Yeats et al., 2004). We hypothesize that the protein might be involved in the proteolytic degradation of extracellular proteins among which FtsL or others that affect the levels of FtsL. However, western blot experiments failed to consistently detect increased amounts of FtsL in a $t s e B$ mutant (not shown). Nevertheless, the short cell phenotype of a $t s e B$ mutant and the septal enrichment is compatible with a role in the division process. The protein is conserved within families belonging to the Bacillales and Lactobacillales orders (STRING database, Szklarczyk et al., 2011). Intriguingly,

\section{References}

Adams, D. W., and Errington, J. (2009). Bacterial cell division: assembly, maintenance and disassembly of the $\mathrm{Z}$ ring. Nat. Rev. Microbiol. 7, 642-653. doi: 10.1038/nrmicro2198

Adams, D. W., Wu, L. J., and Errington, J. (2015). Nucleoid occlusion protein Noc recruits DNA to the bacterial cell membrane. EMBO J. 34, 491-501. doi: $10.15252 / \mathrm{embj} .201490177$

Bi, E. F., and Lutkenhaus, J. (1991). FtsZ ring structure associated with division in Escherichia coli. Nature 354, 161-164. doi: 10.1038/354161a0

Binder, H., and Zschornig, O. (2002). The effect of metal cations on the phase behavior and hydration characteristics of phospholipid membranes. Chem. Phys. Lipids 115, 39-61. doi: 10.1016/S0009-3084(02)00005-1

Bramkamp, M., Weston, L., Daniel, R. A., and Errington, J. (2006). Regulated intramembrane proteolysis of FtsL protein and the control of cell division in Bacillus subtilis. Mol. Microbiol. 62, 580-591. doi: 10.1111/j.13652958.2006.05402.x

Chai, Y., Beauregard, P. B., Vlamakis, H., Losick, R., and Kolter, R. (2012). Galactose metabolism plays a crucial role in biofilm formation by Bacillus subtilis. MBio 3, e00184-e00112. doi: 10.1128/mBio.00184-12

Chien, A. C., Zareh, S. K., Wang, Y. M., and Levin, P. A. (2012). Changes in the oligomerization potential of the division inhibitor UgtP co-ordinate Bacillus subtilis cell size with nutrient availability. Mol. Microbiol. 86, 594-610. doi: $10.1111 / \mathrm{mmi} .12007$

Chopra, I. (1994). Tetracycline analogs whose primary target is not the bacterial ribosome. Antimicrob. Agents Chemother. 38, 637-640. doi: 10.1128/AAC.38.4.637

Chopra, I., and Roberts, M. (2001). Tetracycline antibiotics: mode of action, applications, molecular biology, and epidemiology of bacterial resistance. Microbiol. Mol. Biol. Rev. 65, 232-260. doi: 10.1128/MMBR.65.2.232-2 60.2001 there is a significant co-occurrence in bacterial genomes among TseB, PBP2A and PbpH (STRING database, (Szklarczyk et al., 2011)). These two penicillin-binding proteins are required for cell wall synthesis during elongation (Wei et al., 2003) and were shown to be drivers for MreB dynamics (Dominguez-Escobar et al., 2011; Garner et al., 2011). Possibly, TseB is involved in the switch between septal and lateral cell wall synthesis, which could explain its connection to EzrA.

\section{Acknowledgments}

We thank the members of the Centre for Bacterial Cell Biology for helpful discussions. We are grateful to David Bechhofer for the gift of strain BG239, to Frederico Gueiros-Filho for providing the ZapA-antiserum and to Edward de Koning for help with FtsL western blots. PG was supported by a Marie Curie EST fellowship from the European Commission and by a Biological Sciences Research Council grant (BB/I004238/1) awarded to LH. ER was supported by a short term Marie Curie EST fellowship from the European Commission. LH is supported by NWO grant STW-Vici 12128.

\section{Supplementary Material}

The Supplementary Material for this article can be found online at: http://journal.frontiersin.org/article/10.3389/fmicb. 2015.00346/abstract
Chung, K. M., Hsu, H. H., Yeh, H. Y., and Chang, B. Y. (2007). Mechanism of regulation of prokaryotic tubulin-like GTPase FtsZ by membrane protein EzrA. J. Biol. Chem. 282, 14891-14897. doi: 10.1074/jbc.M605177200

Claessen, D., Emmins, R., Hamoen, L. W., Daniel, R. A., Errington, J., and Edwards, D. H. (2008). Control of the cell elongation-division cycle by shuttling of PBP1 protein in Bacillus subtilis. Mol. Microbiol. 68, 1029-1046. doi: 10.1111/j.1365-2958.2008.06210.x

Cleverley, R. M., Barrett, J. R., Basle, A., Bui, N. K., Hewitt, L., Solovyova, A., et al. (2014). Structure and function of a spectrin-like regulator of bacterial cytokinesis. Nat. Commun. 5, 5421. doi: 10.1038/ncomms6421

Dajkovic, A., Lan, G., Sun, S. X., Wirtz, D., and Lutkenhaus, J. (2008). MinC spatially controls bacterial cytokinesis by antagonizing the scaffolding function of FtsZ. Curr. Biol. 18, 235-244. doi: 10.1016/j.cub.2008.01.042

Daniel, R. A., and Errington, J. (2000). Intrinsic instability of the essential cell division protein FtsL of Bacillus subtilis and a role for DivIB protein in FtsL turnover. Mol. Microbiol. 36, 278-289. doi: 10.1046/j.1365-2958.2000.01857.x

Daniel, R. A., Noirot-Gros, M. F., Noirot, P., and Errington, J. (2006). Multiple interactions between the transmembrane division proteins of Bacillus subtilis and the role of FtsL instability in divisome assembly. J. Bacteriol. 188, 7396-7404. doi: 10.1128/JB.01031-06

Daniel, R. A., Williams, A. M., and Errington, J. (1996). A complex four-gene operon containing essential cell division gene $p b p B$ in Bacillus subtilis. J. Bacteriol. 178, 2343-2350.

Dominguez-Escobar, J., Chastanet, A., Crevenna, A. H., Fromion, V., WedlichSoldner, R., and Carballido-Lopez, R. (2011). Processive movement of MreBassociated cell wall biosynthetic complexes in bacteria. Science 333, 225-228. doi: 10.1126/science.1203466

Duman, R., Ishikawa, S., Celik, I., Strahl, H., Ogasawara, N., Troc, P., et al. (2013). Structural and genetic analyses reveal the protein SepF as a new membrane anchor for the Z ring. Proc. Natl. Acad. Sci. U.S.A. 110, E4601-E4610. doi: $10.1073 /$ pnas. 1313978110 
Estrela, A. I., Pooley, H. M., De Lencastre, H., and Karamata, D. (1991). Genetic and biochemical characterization of Bacillus subtilis 168 mutants specifically blocked in the synthesis of the teichoic acid poly(3-O-beta-Dglucopyranosyl-N-acetylgalactosamine 1-phosphate): gneA, a new locus, is associated with UDP-N-acetylglucosamine 4-epimerase activity. J. Gen. Microbiol. 137, 943-950. doi: 10.1099/00221287-137-4-943

Feucht, A., and Errington, J. (2005). ftsZ mutations affecting cell division frequency, placement and morphology in Bacillus subtilis. Microbiology 151, 2053-2064. doi: 10.1099/mic.0.27899-0

Freymond, P. P., Lazarevic, V., Soldo, B., and Karamata, D. (2006). Poly(glucosyl$\mathrm{N}$-acetylgalactosamine 1-phosphate), a wall teichoic acid of Bacillus subtilis 168: its biosynthetic pathway and mode of attachment to peptidoglycan. Microbiology 152, 1709-1718. doi: 10.1099/mic.0.28814-0

Gamba, P., Veening, J. W., Saunders, N. J., Hamoen, L. W., and Daniel, R. A. (2009). Two-step assembly dynamics of the Bacillus subtilis divisome. J. Bacteriol. 191, 4186-4194. doi: 10.1128/JB.01758-08

Garner, E. C., Bernard, R., Wang, W., Zhuang, X., Rudner, D. Z., and Mitchison, T. (2011). Coupled, circumferential motions of the cell wall synthesis machinery and MreB filaments in B. subtilis. Science 333, 222-225. doi: 10.1126/science. 1203285

Gregory, J. A., Becker, E. C., and Pogliano, K. (2008). Bacillus subtilis MinC destabilizes FtsZ-rings at new cell poles and contributes to the timing of cell division. Genes Dev. 22, 3475-3488. doi: 10.1101/gad.1732408

Gueiros-Filho, F. J., and Losick, R. (2002). A widely conserved bacterial cell division protein that promotes assembly of the tubulin-like protein FtsZ. Genes Dev. 16, 2544-2556. doi: 10.1101/gad.1014102

Gundogdu, M. E., Kawai, Y., Pavlendova, N., Ogasawara, N., Errington, J., Scheffers, D. J., et al. (2011). Large ring polymers align FtsZ polymers for normal septum formation. EMBO J. 30, 617-626. doi: 10.1038/emboj.2010.345

Haeusser, D. P., Schwartz, R. L., Smith, A. M., Oates, M. E., and Levin, P. A. (2004). EzrA prevents aberrant cell division by modulating assembly of the cytoskeletal protein FtsZ. Mol. Microbiol. 52, 801-814. doi: 10.1111/j.13652958.2004.04016.x

Hamoen, L. W., Meile, J. C., De Jong, W., Noirot, P., and Errington, J. (2006). SepF, a novel FtsZ-interacting protein required for a late step in cell division. Mol. Microbiol. 59, 989-999. doi: 10.1111/j.1365-2958.2005.04987.x

Hamoen, L. W., Smits, W. K., De Jong, A., Holsappel, S., and Kuipers, O. P. (2002). Improving the predictive value of the competence transcription factor (ComK) binding site in Bacillus subtilis using a genomic approach. Nucleic Acids Res. 30, 5517-5528. doi: 10.1093/nar/gkf698

Heinrich, J., Lunden, T., Kontinen, V. P., and Wiegert, T. (2008). The Bacillus subtilis $\mathrm{ABC}$ transporter EcsAB influences intramembrane proteolysis through RasP. Microbiology 154, 1989-1997. doi: 10.1099/mic.0.2008/018648-0

Hirokawa, T., Boon-Chieng, S., and Mitaku, S. (1998). SOSUI: classification and secondary structure prediction system for membrane proteins. Bioinformatics 14, 378-379. doi: 10.1093/bioinformatics/14.4.378

Ishikawa, S., Kawai, Y., Hiramatsu, K., Kuwano, M., and Ogasawara, N. (2006). A new FtsZ-interacting protein, YlmF, complements the activity of FtsA during progression of cell division in Bacillus subtilis. Mol. Microbiol. 60, 1364-1380. doi: 10.1111/j.1365-2958.2006.05184.x

Ivanov, D., and Nasmyth, K. (2005). A topological interaction between cohesin rings and a circular minichromosome. Cell 122, 849-860. doi: 10.1016/j.cell.2005.07.018

Kawai, Y., and Ogasawara, N. (2006). Bacillus subtilis EzrA and FtsL synergistically regulate FtsZ ring dynamics during cell division. Microbiology 152, 1129-1141. doi: $10.1099 /$ mic. $0.28497-0$

Krispin, O., and Allmansberger, R. (1998). The Bacillus subtilis galE gene is essential in the presence of glucose and galactose. J. Bacteriol. 180, 2265-2270.

Krulwich, T. A., Jin, J., Guffanti, A. A., and Bechhofer, H. (2001). Functions of tetracycline efflux proteins that do not involve tetracycline. J. Mol. Microbiol. Biotechnol. 3, 237-246.

Le Breton, Y., Mohapatra, N. P., and Haldenwang, W. G. (2006). In vivo random mutagenesis of Bacillus subtilis by use of TnYLB-1, a mariner-based transposon. Appl. Environ. Microbiol. 72, 327-333. doi: 10.1128/AEM.72.1.327333.2006

Levin, P. A., Kurtser, I. G., and Grossman, A. D. (1999). Identification and characterization of a negative regulator of FtsZ ring formation in Bacillus subtilis. Proc. Natl. Acad. Sci. U.S.A. 96, 9642-9647. doi: 10.1073/pnas.96.17.9642
Lewis, P. J., and Marston, A. L. (1999). GFP vectors for controlled expression and dual labelling of protein fusions in Bacillus subtilis. Gene 227, 101-110. doi: 10.1016/S0378-1119(98)00580-0

Lowe, J., and Amos, L. A. (1998). Crystal structure of the bacterial cell-division protein FtsZ. Nature 391, 203-206. doi: 10.1038/34472

Ma, X., and Margolin, W. (1999). Genetic and functional analyses of the conserved C-terminal core domain of Escherichia coli FtsZ. J. Bacteriol. 181, 7531-7544.

Monahan, L. G., Hajduk, I. V., Blaber, S. P., Charles, I. G., and Harry, E. J. (2014). Coordinating bacterial cell division with nutrient availability: a role for glycolysis. MBio 5, e00935-e00914. doi: 10.1128/mBio.00935-14

Morimoto, T., Loh, P. C., Hirai, T., Asai, K., Kobayashi, K., Moriya, S., et al. (2002). Six GTP-binding proteins of the Era/Obg family are essential for cell growth in Bacillus subtilis. Microbiology 148, 3539-3552.

Murray, T., Popham, D. L., and Setlow, P. (1998). Bacillus subtilis cells lacking penicillin-binding protein 1 require increased levels of divalent cations for growth. J. Bacteriol. 180, 4555-4563.

Nelson, M. L. (1998). Chemical and biological dynamics of tetracyclines. Adv. Dent. Res. 12, 5-11. doi: 10.1177/08959374980120011901

Nicolas, P., Mader, U., Dervyn, E., Rochat, T., Leduc, A., Pigeonneau, N., et al. (2012). Condition-dependent transcriptome reveals high-level regulatory architecture in Bacillus subtilis. Science 335, 1103-1106. doi: 10.1126/science. 1206848

Oliva, B., and Chopra, I. (1992). Tet determinants provide poor protection against some tetracyclines: further evidence for division of tetracyclines into two classes. Antimicrob. Agents Chemother. 36, 876-878. doi: 10.1128/AAC.36.4.876

Pacheco-Gomez, R., Cheng, X., Hicks, M. R., Smith, C. J., Roper, D. I., Addinall, S., et al. (2013). Tetramerization of ZapA is required for FtsZ bundling. Biochem. J. 449, 795-802. doi: 10.1042/BJ20120140

Peters, P. C., Migocki, M. D., Thoni, C., and Harry, E. J. (2007). A new assembly pathway for the cytokinetic $\mathrm{Z}$ ring from a dynamic helical structure in vegetatively growing cells of Bacillus subtilis. Mol. Microbiol. 64, 487-499. doi: 10.1111/j.1365-2958.2007.05673.x

Robson, S. A., Michie, K. A., Mackay, J. P., Harry, E., and King, G. F. (2002). The Bacillus subtilis cell division proteins FtsL and DivIC are intrinsically unstable and do not interact with one another in the absence of other septasomal components. Mol. Microbiol. 44, 663-674. doi: 10.1046/j.1365-2958.2002.02920.x

Schirner, K., Marles-Wright, J., Lewis, R. J., and Errington, J. (2009). Distinct and essential morphogenic functions for wall- and lipo-teichoic acids in Bacillus subtilis. EMBO J. 28, 830-842. doi: 10.1038/emboj.2009.25

Singh, J. K., Makde, R. D., Kumar, V., and Panda, D. (2007). A membrane protein, EzrA, regulates assembly dynamics of FtsZ by interacting with the C-terminal tail of FtsZ. Biochemistry 46, 11013-11022. doi: 10.1021/bi700710j

Singh, J. K., Makde, R. D., Kumar, V., and Panda, D. (2008). SepF increases the assembly and bundling of FtsZ polymers and stabilizes FtsZ protofilaments by binding along its length. J. Biol. Chem. 283, 31116-31124. doi: 10.1074/jbc.M805910200

Soldo, B., Scotti, C., Karamata, D., and Lazarevic, V. (2003). The Bacillus subtilis Gne (GneA, GalE) protein can catalyse UDP-glucose as well as UDP$\mathrm{N}$-acetylglucosamine 4-epimerisation. Gene 319, 65-69. doi: 10.1016/S03781119(03)00793-5

Strahl, H., and Hamoen, L. W. (2010). Membrane potential is important for bacterial cell division. Proc. Natl. Acad. Sci. U.S.A. 107, 12281-12286. doi: 10.1073/pnas.1005485107

Surdova, K., Gamba, P., Claessen, D., Siersma, T., Jonker, M. J., Errington, J., et al. (2013). The conserved DNA-binding protein WhiA is involved in cell division in Bacillus subtilis. J. Bacteriol. 195, 5450-5460. doi: 10.1128/JB.00507-13

Szklarczyk, D., Franceschini, A., Kuhn, M., Simonovic, M., Roth, A., Minguez, P., et al. (2011). The STRING database in 2011: functional interaction networks of proteins, globally integrated and scored. Nucleic Acids Res. 39, D561-D568. doi: 10.1093/nar/gkq973

Tavares, J. R., De Souza, R. F., Meira, G. L., and Gueiros-Filho, F. J. (2008). Cytological characterization of YpsB, a novel component of the Bacillus subtilis divisome. J. Bacteriol. 190, 7096-7107. doi: 10.1128/JB. 00064-08

Trieu-Cuot, P., and Courvalin, P. (1983). Nucleotide sequence of the Streptococcus faecalis plasmid gene encoding the $3^{\prime} 5^{\prime \prime}$-aminoglycoside phosphotransferase type III. Gene 23, 331-341. doi: 10.1016/0378-1119(83) 90022-7 
van Baarle, S., and Bramkamp, M. (2010). The MinCDJ system in Bacillus subtilis prevents minicell formation by promoting divisome disassembly. PLoS ONE 5:e9850. doi: 10.1371/journal.pone.0009850

Vest, R. S., Gonzales, L. J., Permann, S. A., Spencer, E., Hansen, L. D., Judd, A. M., et al. (2004). Divalent cations increase lipid order in erythrocytes and susceptibility to secretory phospholipase A2. Biophys. J. 86, 2251-2260. doi: 10.1016/S0006-3495(04)74283-6

Vincent, M., England, L. S., and Trevors, J. T. (2004). Cytoplasmic membrane polarization in Gram-positive and Gram-negative bacteria grown in the absence and presence of tetracycline. Biochim. Biophys. Acta 1672, 131-134. doi: 10.1016/j.bbagen.2004.03.005

Wadenpohl, I., and Bramkamp, M. (2010). DivIC stabilizes FtsL against RasP cleavage. J. Bacteriol. 192, 5260-5263. doi: 10.1128/JB. 00287-10

Wang, X., Huang, J., Mukherjee, A., Cao, C., and Lutkenhaus, J. (1997). Analysis of the interaction of FtsZ with itself, GTP, and FtsA. J. Bacteriol. 179, 5551-5559.

Weart, R. B., Lee, A. H., Chien, A. C., Haeusser, D. P., Hill, N. S., and Levin, P. A. (2007). A metabolic sensor governing cell size in bacteria. Cell 130, 335-347. doi: $10.1016 /$ j.cell.2007.05.043

Wei, Y., and Bechhofer, D. H. (2002). Tetracycline induces stabilization of mRNA in Bacillus subtilis. J. Bacteriol. 184, 889-894. doi: 10.1128/jb.184.4.889894.2002

Wei, Y., Havasy, T., McPherson, D. C., and Popham, D. L. (2003). Rod shape determination by the Bacillus subtilis class B penicillin-binding proteins encoded by $p b p A$ and $p b p H$. J. Bacteriol. 185, 4717-4726. doi: 10.1128/JB.185.16.47174726.2003
Weidenmaier, C., and Peschel, A. (2008). Teichoic acids and related cell-wall glycopolymers in Gram-positive physiology and host interactions. Nat. Rev. Microbiol. 6, 276-287. doi: 10.1038/nrmicro1861

Williams, G., and Smith, I. (1979). Chromosomal mutations causing resistance to tetracycline in Bacillus subtilis. Mol. Gen. Genet. 177, 23-29. doi: 10.1007/BF00267249

Wu, L. J., and Errington, J. (2004). Coordination of cell division and chromosome segregation by a nucleoid occlusion protein in Bacillus subtilis. Cell 117, 915-925. doi: 10.1016/j.cell.2004.06.002

Wu, L. J., Ishikawa, S., Kawai, Y., Oshima, T., Ogasawara, N., and Errington, J. (2009). Noc protein binds to specific DNA sequences to coordinate cell division with chromosome segregation. EMBO J. 28, 1940-1952. doi: 10.1038/emboj.2009.144

Yeats, C., Rawlings, N. D., and Bateman, A. (2004). The PepSY domain: a regulator of peptidase activity in the microbial environment? Trends Biochem. Sci. 29 169-172. doi: 10.1016/j.tibs.2004.02.004

Conflict of Interest Statement: The authors declare that the research was conducted in the absence of any commercial or financial relationships that could be construed as a potential conflict of interest.

Copyright (c) 2015 Gamba, Rietkötter, Daniel and Hamoen. This is an open-access article distributed under the terms of the Creative Commons Attribution License (CC $B Y)$. The use, distribution or reproduction in other forums is permitted, provided the original author(s) or licensor are credited and that the original publication in this journal is cited, in accordance with accepted academic practice. No use, distribution or reproduction is permitted which does not comply with these terms. 